\title{
Factorizable $R$-Matrices for Small Quantum Groups
}

Simon LENTNER and Tobias OHRMANN

Fachbereich Mathematik, University of Hamburg, Bundesstraße 55, 20146 Hamburg, Germany

E-mail:simon.lentner@uni-hamburg.de,tobias.ohrmann@uni-hamburg.de

Received January 16, 2017, in final form September 15, 2017; Published online September 25, 2017 https://doi.org/10.3842/SIGMA.2017.076

\begin{abstract}
Representations of small quantum groups $u_{q}(\mathfrak{g})$ at a root of unity and their extensions provide interesting tensor categories, that appear in different areas of algebra and mathematical physics. There is an ansatz by Lusztig to endow these categories with the structure of a braided tensor category. In this article we determine all solutions to this ansatz that lead to a non-degenerate braiding. Particularly interesting are cases where the order of $q$ has common divisors with root lengths. In this way we produce familiar and unfamiliar series of (non-semisimple) modular tensor categories. In the degenerate cases we determine the group of so-called transparent objects for further use.
\end{abstract}

Key words: factorizable; $R$-matrix; quantum group; modular tensor category; transparent object

2010 Mathematics Subject Classification: 17B37; 20G42; 81R50; 18D10

\section{Introduction}

Hopf algebras with $R$-matrices, so called quasitriangular Hopf algebras, give rise to tensor categories with a braiding $c: V \otimes W \stackrel{\sim}{\longrightarrow} W \otimes V$. Of particular interest are braided tensor categories where the braiding fulfills a certain non-degeneracy condition, see Definition 5.1, which is equivalent to the fact that there are no transparent objects $V$, i.e., no objects where the double-braiding $c^{2}: V \otimes W \stackrel{\sim}{\longrightarrow} V \otimes W$ is the identity for all $W$. A $\mathbb{C}$-linear tensor category with a nondegenerate braiding, as well as finiteness conditions and another natural transformation $\theta: V \stackrel{\sim}{\longrightarrow} V$ (twist), is called a modular tensor category. Note that we do not require the category to be semisimple.

Modular tensor categories have many interesting applications: They give rise to topological invariants and mapping class group actions [7, 19]. For example, the standard generators $T, S$ of the mapping class group of the torus $\mathrm{SL}_{2}(\mathbb{Z})$ are constructed from $\theta$ and $c^{2}$, respectively. A different source for modular tensor categories in mathematical physics are vertex algebras. There are only few example classes of modular tensor categories, in particular non-semisimple ones.

The aim of the present article is to provide modular tensor categories from small quantum groups $u_{q}(\mathfrak{g})$ at a primitive $\ell$-th root of unity $q$ for a finite-dimensional simple complex Lie algebra $\mathfrak{g}$. Lusztig [12] has constructed these finite-dimensional Hopf algebras and provided an ansatz for an $R$-matrix $R_{0} \bar{\Theta}$, where the fixed element $\bar{\Theta} \in u_{q}(\mathfrak{g})^{-} \otimes u_{q}(\mathfrak{g})^{+}$is constructed from a dual basis of PBW generators, while $R_{0} \in u_{q}(\mathfrak{g})^{0} \otimes u_{q}(\mathfrak{g})^{0}$ is a free parameter subject to some constraints. He gives one canonical solution for $R_{0}$ whenever $\ell$ has no common divisors with root lengths, otherwise there are cases where no $R$-matrix exists [8] and the quantum group becomes more interesting [9], involving, e.g., the dual root system. Of particular interest in conformal field theory $[4,5,6]$ is the most extreme case where all root lengths $(\alpha, \alpha)$ divide $\ell$. In particular our article addresses the question, which modular tensor category appear in these cases. We find indeed, e.g., in Lemma 4.7 that these extremal cases give especially nice $R$-matrices; although in general they are not factorizable and will require modularization (see for example [2]) to match the CFT side. 
But even if there are no common divisors with the root length, the resulting braided tensor categories may not fulfill the non-degeneracy condition and hence provides no modular tensor category.

Both obstacles (existence and non-degeneracy) can be be resolved by extending the Cartan part of the quantum group by a choice of a lattice $\Lambda_{R} \subseteq \Lambda \subseteq \Lambda_{W}$ between root- and weight-lattice, respectively a choice of a subgroup of the fundamental group $\pi_{1}:=\Lambda_{W} / \Lambda_{R}$, corresponding to a choice of a Lie group between adjoint and simply-connected form. These extensions are already present in [12] as the choice of two lattices $X, Y$ with pairing $X \times Y \rightarrow \mathbb{C}^{\times}$ (root datum). In this way the number of possible $R$ matrices increases and the purpose of our paper is to study them all.

In a previous article [11] we have already constructed some solutions $R_{0}$ in this spirit (under some additional assumptions). As it turns out, the solutions can be parametrized by subgroups $H_{1}, H_{2} \subset \pi_{1}$ and group pairings between $H_{1}, H_{2}$, and the set of solutions depends on the common divisors of $\ell$ not only with root lengths, but also divisors of the Cartan matrix. Some cases admit no braided structure, while others have multiple in-equivalent solutions. An interesting occurrence was for example that $B_{n}$ behaves differently for $n$ odd or even, and that $D_{2 n}$ with non-cyclic fundamental group allows several more solutions with non-symmetric $R_{0}$.

In the present article we conclude this effort: First we introduce more systematical techniques that allow us to compute a list of all quasitriangular structures (without additional assumptions, so we find more solutions). Then our new techniques allow us to determine, which of these choices fulfill the non-degeneracy condition. We also determine which cases have a ribbon structure. A main role in the first part is played by a natural pairing $a_{\ell}$ on the fundamental group $\pi_{1}$ which depends only on the common divisors of $\ell$ with the fundamental group and encapsulates the essential $\ell$-dependence. Then the non-degeneracy of the braiding turns out to depend only on the 2-torsion of the abelian group in question.

Our result produces a list of modular tensor categories for representations of quantum groups. Moreover we use our methods to explicitly describe the group of transparent objects if the category is not modular, which is for example a prerequisite for modularization.

We now discuss our methods and results in more detail:

In Section 2 we briefly recall the Lie theory and Hopf algebra preliminaries: For every finitedimensional (semi-)simple complex Lie algebra $\mathfrak{g}$ and a primitive $\ell$-th root of unity $q$ Lusztig has introduced in [12] the small quantum group $u_{q}(\mathfrak{g})$ which has a triangular decomposition $u_{q}^{+} u_{q}^{0} u_{q}^{-}$ where the (exponentiated) Cartan algebra $u_{q}^{0}$ is the groupring of the root lattice $\Lambda_{R}$ modulo some suitable sublattice and $u_{q}^{ \pm}$are generated by simple root vectors $E_{\alpha_{i}}, F_{\alpha_{i}}$ fulfilling $q$-deformed Serre relations. In [13, Section 32] he gives an ansatz for an $R$-matrix in the form $R_{0} \bar{\Theta}$, where $\bar{\Theta}$ consists of dual PBW basis' and $R_{0} \in u_{q}^{0} \otimes u_{q}^{0}$ is an arbitrary element in the Cartan part that has to fulfill certain relations.

Our goal is to study the existence and non-degeneracy of $R$-matrices of this form for the quantum group $u_{q}\left(\mathfrak{g}, \Lambda, \Lambda^{\prime}\right)$ with any choice of lattice between root- and weight-lattice $\Lambda_{R} \subseteq \Lambda \subseteq \Lambda_{W}$ and any possible choice of quotient by a subgroup $\Lambda^{\prime} \subseteq \Lambda$ in the Cartan part $u^{0}=\mathbb{C}\left[\Lambda / \Lambda^{\prime}\right]$. Later, we prove that $\Lambda^{\prime}$ is in fact unique if we want a quasitriagular structure (Corollary 3.6).

The $R_{0}$-matrix has the following interpretation: It is an $R$-matrix for the groupring $\mathbb{C}\left[\Lambda / \Lambda^{\prime}\right]$ and it appears as the braiding between highest-weight vectors in our $u_{q}(\mathfrak{g})$-modules. Thus the previous theorem clarifies which choices for an $R$-matrix for the group ring lift to the quantum group.

In Section 3 we address the question of constructing quasi-triangular $R$-matrices. First we briefly recall the following general combinatorial result in [11]:

Theorem 3.3. The $R_{0}$-matrix is necessarily of the form

$$
f(\mu, \nu)=\frac{1}{d} q^{-(\mu, \nu)} g(\bar{\mu}, \bar{\nu}) \delta_{\bar{\mu} \in H_{1}} \delta_{\bar{\nu} \in H_{2}},
$$


where $H_{1}, H_{2}$ are subgroups of $\Lambda / \Lambda_{R} \subseteq \pi_{1}$ with $\left|H_{1}\right|=\left|H_{2}\right|=: d$ (not necessarily isomorphic!) and $g: H_{1} \times H_{2} \rightarrow \mathbb{C}^{\times}$is a pairing of groups.

Then we proceed differently than in the previous article: Using the previous result, we prove in Lemma 3.5 that the quasitriangularity of $R$ is equivalent to the assertion that the group pairing $\hat{f}:=\left|\Lambda / \Lambda^{\prime}\right| \cdot f$ between the preimages $G_{i}:=\Lambda_{i} / \Lambda^{\prime}$ of the groups $H_{i}$ is non-degenerate (which is no surprise). In particular we show that this condition fixes $\Lambda^{\prime}$ uniquely. In later applications we often encounter $\hat{f}$ as a natural identification of $G_{1}$ and the dual $\hat{G}_{2}$, e.g., when studying representation theory.

To find all solutions $f$ with this property we develop a machinery to push $\hat{f}$ into the fundamental group $\pi_{1}$, which encapsulates all the $\ell$-dependence: In Definition 3.8 we give an abstract characterization of a centralizer transfer map

$$
A_{\ell}: \Lambda / \Lambda_{R} \stackrel{\sim}{\longrightarrow} \operatorname{Cent}_{\Lambda}^{\ell}\left(\Lambda_{R}\right) / \operatorname{Cent}_{\Lambda_{R}}^{\ell}(\Lambda)
$$

(without proving that it always exists). In a generic case this is just multiplication by $\ell$, but it severely depends on common divisors of $\ell$ with root length and divisors of the Cartan matrix. With this matrix we can transfer $q^{-(\mu, \nu)}$ to a natural form $a_{g}^{\ell}$ on the fundamental group. We prove that $\hat{f}$ is non-degenerate iff $a_{g}^{\ell}(\mu, \nu)=q^{-\left(\mu, A_{\ell}(\nu)\right)} \cdot g\left(\mu, A_{\ell}(\nu)\right)$ is non-degenerate. This explains why the set of solutions, say for fundamental group $\mathbb{Z}_{n}$ always looks like the subset of invertible elements $\mathbb{Z}_{n}^{\times}$but it is shifted (namely by $A_{\ell}$ ) depending on $\ell$ and the root system in question.

In Section 4 the remaining computational work is done for quasitriagularity: We calculate a list containing $a_{g}^{\ell}$ for all simple $\mathfrak{g}$, depending on common divisors of $\ell$ with root length and divisors. We thus write down all solutions for $f$ and hence $R$-matrices. The calculation starts with the Smith normal form for the Cartan matrix in question and uses three cases: For $\Lambda=\Lambda_{W}$ we have a generic construction, the cases $A_{n}$ with their large fundamental group $\mathbb{Z}_{n+1}$ is treated by hand, as is $D_{2 n}$ with non-cyclic fundamental group, which has the only cases allowing $\Lambda_{1} \neq \Lambda_{2}$.

In Section 5 we address our main issue of factorizability with our new tools:

In Section 5.1 we introduce factorizability. Then we calculate the monodromy matrix $R_{21} R$ for an arbitrary choice of $R$-matrix in terms the $R_{0}$-part. This gives a purely lattice theoretic problem equivalent to the factorizability of such an $R$-matrix. Then we prove in the main Theorem 5.9 that factorizability is equivalent to the non-degeneracy of a symmetrization $\operatorname{Sym}_{G}(\hat{f})$ of $\hat{f}$. As will turn out later, the radical of this form is isomorphic to the group of transparent objects.

In Section 5.2 we restrict ourselves to the symmetric case where $H_{1}=H_{2}$ and $f, g$ are symmetric. Other cases appear only in some of the non-cyclic $\mathbb{Z}_{2} \times \mathbb{Z}_{2}$-extension for type $\mathfrak{g}=D_{2 n}$ and are dealt with in Section 5.3 and give surprising new solutions.

The main result for the symmetric case is that the radical of the form $\operatorname{Sym}_{G}(\hat{f})$ is in this case simply the 2-torsion of $\Lambda / \Lambda^{\prime}$ (Example 5.11) and that this is non-degenerate precisely for odd $\ell$ and odd $\Lambda / \Lambda_{R}$ as well as for $\mathfrak{g}=B_{n}, \Lambda=\Lambda_{R}, \ell \equiv 2 \bmod 4$ including $A_{1}$.

In Section 5.4 we prove the following result:

Lemma 5.19. The transparent objects in the category of representations of the Hopf algebra $u_{q}(\mathfrak{g}, \Lambda)$ with $R$-matrix given by Lusztig's ansatz are 1 -dimensional objects $\mathbb{C}_{\chi}$ and are the $f$ transformed of the radical of $\operatorname{Sym}_{G}(\hat{f})$ :

$$
\chi(\mu)=f(\mu, \xi), \quad \xi \in \operatorname{Rad}\left(\operatorname{Sym}_{G}(\hat{f})\right) .
$$

In the following we summarize our results by a table containing all quasitriangular quantum groups $u_{q}(\mathfrak{g}, \Lambda)$ with their group of transparent objects. In Section 6 we show that all our quasitriangular quantum groups admit a ribbon element. The factorizable solutions and thus modular tensor categories are $\ell$ odd, $\Lambda=\Lambda_{R}$ and the following new factorizable cases: 
$\left(\ell\right.$ odd, $\left.E_{6}, \Lambda=\Lambda_{W}\right)$ and $\left(\ell \equiv 2 \bmod 4, \mathfrak{g}=B_{n}, \Lambda=\Lambda_{R}\right)$ (including $\left.A_{1}\right)$ and $(\ell$ odd, $\mathfrak{g}=D_{2 n}, \Lambda_{1} \neq \Lambda_{2}$ ). All other cases can be modularized as discussed in Question 7.3.

The columns of the following table are labeled by

1) the finite-dimensional simple complex Lie algebra $\mathfrak{g}$,

2) the natural number $\ell$, determining the root of unity $q=\exp \left(\frac{2 \pi i}{\ell}\right)$,

3 ) the number of possible $R$-matrices for the Lusztig ansatz,

4) the subgroups $H_{i} \subseteq H=\Lambda / \Lambda_{R}$ introduced in Theorem 3.3,

5) the subgroups $H_{i}$ in terms of generators given by multiples of fundamental dominant weights $\lambda_{i} \in \Lambda_{W}$,

6) the group pairing $g: H_{1} \times H_{2} \rightarrow \mathbb{C}^{\times}$determined by its values on generators,

7) the group of transparent objects $T \subseteq \Lambda / \Lambda^{\prime}$ introduced in Lemma 5.19.

\begin{tabular}{|c|c|c|c|c|c|c|}
\hline $\mathfrak{g}$ & $\ell$ & $\#$ & $H_{i} \cong$ & $H_{i}(i=1,2)$ & $g$ & $T \subseteq \Lambda / \Lambda^{\prime}$ \\
\hline \multirow{2}{*}{ all } & $\ell$ odd & 1 & \multirow{2}{*}{$\mathbb{Z}_{1}$} & \multirow{2}{*}{$\langle 0\rangle$} & \multirow{2}{*}{$g=1$} & $\mathbf{0}$ \\
\hline & $\ell \equiv 0 \bmod 4$ & 1 & & & & $\mathbb{Z}_{2}^{n}$ \\
\hline $\begin{array}{c}A_{n \geq 1} \\
\pi_{1}=\mathbb{Z}_{n+1}\end{array}$ & & $\infty$ & $\begin{array}{c}\mathbb{Z}_{d} \\
d \mid n+1\end{array}$ & $\begin{array}{c}\left\langle\hat{d} \lambda_{n}\right\rangle \\
\hat{d}=\frac{n+1}{d}\end{array}$ & $\begin{array}{c}g\left(\hat{d} \lambda_{n}, \hat{d} \lambda_{n}\right)=\exp \left(\frac{2 \pi i k}{d}\right) \\
\operatorname{gcd}\left(d, \frac{k \ell-\hat{d} n}{\operatorname{gcd}(\ell, \hat{d})}\right)=1\end{array}$ & $\begin{array}{c}\mathbb{Z}_{2}^{n-1}, 2 \nmid x \\
\mathbb{Z}_{2}^{n}, 2 \mid x \\
x=\frac{d \ell}{\operatorname{gcd}(\ell, \hat{d})}\end{array}$ \\
\hline \multirow{4}{*}{$\begin{array}{c}B_{n \geq 2} \\
\pi_{1}=\mathbb{Z}_{2}\end{array}$} & $\ell \equiv 2 \bmod 4$ & 1 & $\mathbb{Z}_{1}$ & $\langle 0\rangle$ & $g=1$ & $\mathbf{0}$ \\
\hline & $\ell \equiv 2 \bmod 4$ & 2 & $\mathbb{Z}_{2}$ & $\left\langle\lambda_{n}\right\rangle$ & $g\left(\lambda_{n}, \lambda_{n}\right)= \pm 1$ & $\mathbb{Z}_{2}$ \\
\hline & $\ell \equiv 0 \bmod 4$ & 2 & $\mathbb{Z}_{2}$ & $\left\langle\lambda_{n}\right\rangle$ & $g\left(\lambda_{n}, \lambda_{n}\right)= \pm 1$ & $\mathbb{Z}_{2}^{n}$ \\
\hline & $\ell$ odd & 1 & $\mathbb{Z}_{2}$ & $\left\langle\lambda_{n}\right\rangle$ & $g\left(\lambda_{n}, \lambda_{n}\right)=(-1)^{n+1}$ & $\mathbb{Z}_{2}$ \\
\hline \multirow{4}{*}{$\begin{array}{c}C_{n \geq 3} \\
\pi_{1}=\mathbb{Z}_{2}\end{array}$} & $\ell \equiv 2 \bmod 4$ & 1 & $\mathbb{Z}_{1}$ & $\langle 0\rangle$ & $g=1$ & $\mathbb{Z}_{2}^{n-2}$ \\
\hline & $\ell \equiv 2 \bmod 4$ & 1 & \multirow{3}{*}{$\mathbb{Z}_{2}$} & \multirow{3}{*}{$\left\langle\lambda_{n}\right\rangle$} & $g\left(\lambda_{n}, \lambda_{n}\right)=1$ & $\mathbb{Z}_{2}^{n-1}$ \\
\hline & $\ell \equiv 0 \bmod 4$ & 2 & & & $g\left(\lambda_{n}, \lambda_{n}\right)= \pm 1$ & $\mathbb{Z}_{2}^{n}$ \\
\hline & $\ell$ odd & 1 & & & $g\left(\lambda_{n}, \lambda_{n}\right)=-1$ & $\mathbb{Z}_{2}$ \\
\hline \multirow{10}{*}{$\begin{array}{c}D_{2 n \geq 4} \\
\pi_{1}=\mathbb{Z}_{2} \times \mathbb{Z}_{2}\end{array}$} & $\ell \equiv 2 \bmod 4$ & 1 & $\mathbb{Z}_{1}$ & $\langle 0\rangle$ & $g=1$ & $\mathbb{Z}_{2}^{2(n-1)}$ \\
\hline & $\ell \equiv 2 \bmod 4$ & 1 & \multirow{3}{*}{$\mathbb{Z}_{2}$} & \multirow[t]{3}{*}{$H_{1} \cong\left\langle\lambda_{2 n-1}\right\rangle$} & $g\left(\lambda_{2 n-1}, \lambda_{2 n}\right)=(-1)^{n}$ & \multirow{2}{*}{$\mathbb{Z}_{2}^{2 n}$} \\
\hline & $\ell \equiv 0 \bmod 4$ & $2 \delta_{2 \mid n}$ & & & $g\left(\lambda_{2 n-1}, \lambda_{2 n}\right)= \pm 1, n$ even & \\
\hline & $\ell$ odd & 1 & & & $g\left(\lambda_{2 n-1}, \lambda_{2 n}\right)=-1$ & $\mathbf{0}$ \\
\hline & $\ell \equiv 2 \bmod 4$ & 1 & \multirow{3}{*}{$\mathbb{Z}_{2}$} & \multirow{3}{*}{$\left\langle\lambda_{2 n}\right\rangle$} & $g\left(\lambda_{2 n}, \lambda_{2 n}\right)=(-1)^{n+1}$ & $\mathbb{Z}_{2}^{2 n-1}$ \\
\hline & $\ell \equiv 0 \bmod 4$ & $2 \delta_{2 \nmid n}$ & & & $g\left(\lambda_{2 n}, \lambda_{2 n}\right)= \pm 1, n$ odd & $\mathbb{Z}_{2}^{2 n}$ \\
\hline & $\ell$ odd & 1 & & & $g\left(\lambda_{2 n}, \lambda_{2 n}\right)=-1$ & $\mathbb{Z}_{2}$ \\
\hline & $\ell$ even & 16 & \multirow{3}{*}{$\mathbb{Z}_{2} \times \mathbb{Z}_{2}$} & \multirow{3}{*}{$\left\langle\lambda_{2 n}, \lambda_{2 n+1}\right\rangle$} & $g\left(\lambda_{2(n-1)+i}, \lambda_{2(n-1)+j}\right)= \pm 1$ & $\mathbb{Z}_{2}^{2 n}$ \\
\hline & \multirow{2}{*}{$\ell$ odd } & \multirow{2}{*}{6} & & & $\operatorname{det}(K)=K_{12}+K_{21}=0 \bmod 2$ & $\mathbb{Z}_{2}$ \\
\hline & & & & & $\operatorname{det}(K)=K_{12}+K_{21}=1 \bmod 2$ & $\mathbb{Z}_{2}^{2}$ \\
\hline
\end{tabular}




\begin{tabular}{|c|c|c|c|c|c|c|}
\hline \multirow{6}{*}{$\begin{array}{c}D_{2 n+1 \geq 5} \\
\pi_{1}=\mathbb{Z}_{4}\end{array}$} & $\ell \equiv 2 \bmod 4$ & 1 & $\mathbb{Z}_{1}$ & $\langle 0\rangle$ & $g=1$ & $\mathbb{Z}_{2}^{2 n}$ \\
\hline & $\ell \equiv 2 \bmod 4$ & 1 & \multirow{3}{*}{$\mathbb{Z}_{2}$} & \multirow{3}{*}{$\left\langle 2 \lambda_{2 n+1}\right\rangle$} & $g\left(2 \lambda_{2 n+1}, 2 \lambda_{2 n+1}\right)=1$ & \multirow{2}{*}{$\mathbb{Z}_{2}^{2 n+1}$} \\
\hline & $\ell \equiv 0 \bmod 4$ & 2 & & & $g\left(2 \lambda_{2 n+1}, 2 \lambda_{2 n+1}\right)= \pm 1$ & \\
\hline & $\ell$ odd & 1 & & & $g\left(2 \lambda_{2 n+1}, 2 \lambda_{2 n+1}\right)=-1$ & $\mathbb{Z}_{2}$ \\
\hline & $\ell$ even & 4 & \multirow{2}{*}{$\mathbb{Z}_{4}$} & \multirow{2}{*}{$\left\langle\lambda_{2 n+1}\right\rangle$} & $g\left(\lambda_{2 n+1}, \lambda_{2 n+1}\right)=c, c^{4}=1$ & $\mathbb{Z}_{2}^{2 n+1}$ \\
\hline & $\ell$ odd & 2 & & & $g\left(\lambda_{2 n+1}, \lambda_{2 n+1}\right)= \pm 1$ & $\mathbb{Z}_{2}$ \\
\hline \multirow{4}{*}{$\begin{array}{c}E_{6} \\
\pi_{1}=\mathbb{Z}_{3}\end{array}$} & $\ell \equiv 2 \bmod 4$ & 1 & $\mathbb{Z}_{1}$ & $\langle 0\rangle$ & $g=1$ & $\mathbb{Z}_{2}^{6}$ \\
\hline & $\ell \equiv 0 \bmod 3$ & 3 & \multirow{3}{*}{$\mathbb{Z}_{3}$} & \multirow{3}{*}{$\left\langle\lambda_{n}\right\rangle$} & $g\left(\lambda_{n}, \lambda_{n}\right)=c, c^{3}=1$ & \multirow{2}{*}{$\mathbb{Z}_{2}^{6}, 2 \mid \ell$} \\
\hline & $\ell \equiv 1 \bmod 3$ & 2 & & & $g\left(\lambda_{n}, \lambda_{n}\right)=1, \exp \left(\frac{2 \pi i 2}{3}\right)$ & \\
\hline & $\ell \equiv 2 \bmod 3$ & 2 & & & $g\left(\lambda_{n}, \lambda_{n}\right)=1, \exp \left(\frac{2 \pi i}{3}\right)$ & $\mathbf{0}, 2 \nmid \ell$ \\
\hline \multirow{3}{*}{$\begin{array}{c}E_{7} \\
\pi_{1}=\mathbb{Z}_{2}\end{array}$} & $\ell \equiv 2 \bmod 4$ & 1 & $\mathbb{Z}_{1}$ & $\langle 0\rangle$ & $g=1$ & $\mathbb{Z}_{2}^{6}$ \\
\hline & $\ell$ even & 2 & \multirow{2}{*}{$\mathbb{Z}_{2}$} & \multirow{2}{*}{$\left\langle\lambda_{n}\right\rangle$} & $g\left(\lambda_{n}, \lambda_{n}\right)= \pm 1$ & $\mathbb{Z}_{2}^{n}$ \\
\hline & $\ell$ odd & 1 & & & $g\left(\lambda_{n}, \lambda_{n}\right)=1$ & $\mathbb{Z}_{2}$ \\
\hline$E_{8}$ & $\ell \equiv 2 \bmod 4$ & 1 & $\mathbb{Z}_{1}$ & $\langle 0\rangle$ & $g=1$ & $\mathbb{Z}_{2}^{8}$ \\
\hline$F_{4}$ & $\ell \equiv 2 \bmod 4$ & 1 & $\mathbb{Z}_{1}$ & $\langle 0\rangle$ & $g=1$ & $\mathbb{Z}_{2}^{2}$ \\
\hline$G_{2}$ & $\ell \equiv 2 \bmod 4$ & 1 & $\mathbb{Z}_{1}$ & $\langle 0\rangle$ & $g=1$ & $\mathbb{Z}_{2}^{2}$ \\
\hline
\end{tabular}

Table 1: Solutions for $R_{0}$-matrices.

In the case $D_{2 n}, \Lambda=\Lambda_{W}, g$ is uniquely defined by a $(2 \times 2)$-matrix $K \in \mathfrak{g l}\left(2, \mathbb{F}_{2}\right)$, s.t. $g\left(\lambda_{2(n-1)+i}, \lambda_{2(n-1)+j}\right)=\exp \left(\frac{2 \pi i K_{i j}^{g}}{2}\right)$ for $i, j \in\{1,2\}$.

\section{Preliminaries}

\subsection{Lie theory}

Throughout this article, $\mathfrak{g}$ denotes a finite-dimensional simple complex Lie algebra. We fix a choice of simple roots $\Delta=\left\{\alpha_{i} \mid i \in I\right\}$, so that the Cartan matrix $C$ is given by $C_{i j}=2 \frac{\left(\alpha_{i}, \alpha_{j}\right)}{\left(\alpha_{i}, \alpha_{i}\right)}$, where $($,$) denotes the normalized Killing form. For a root \alpha$, we define $d_{\alpha}:=\frac{(\alpha, \alpha)}{2}$ and set $d_{i}=d_{\alpha_{i}}$. By $\Lambda_{R}:=\mathbb{Z}[\Delta]$ and $\Lambda_{R}^{\vee}:=\mathbb{Z}\left[\Delta^{\vee}\right]$ we denote the (co)root lattice of $\mathfrak{g}$.

By $\Lambda_{W}$, we denote the weight lattice spanned by fundamental dominant weights $\lambda_{i}$, which are defined by the equation $\left(\lambda_{i}, \alpha_{j}\right)=\delta_{i, j} d_{i}$. Finally, we define the co-weight lattice $\Lambda_{W}^{\vee}$ as the $\mathbb{Z}$ span of the elements $\lambda_{i}^{\vee}:=\frac{\lambda_{i}}{d_{i}}$. The quotient $\pi_{1}:=\Lambda_{W} / \Lambda_{R}$ is called the fundamental group of $\mathfrak{g}$.

One can easily see that the Killing form restricts to a perfect pairing (, ): $\Lambda_{W}^{\vee} \times \Lambda_{R} \rightarrow \mathbb{Z}$ and that we get a string of inclusions $\Lambda_{R} \subseteq \Lambda_{R}^{\vee} \subseteq \Lambda_{W} \subseteq \Lambda_{W}^{\vee}$.

\subsection{Lusztig's ansatz for $R$-matrices}

The starting point for our work [11] was Lusztig's ansatz in [13, Section 32.1] for a universal $R$ matrix of $U_{q}(\mathfrak{g})$. Namely, for a specific element $\bar{\Theta} \in U_{\bar{q}}^{\geq 0} \otimes U_{q}^{\leq 0}$ from a dual basis and a suitable (not further specified) element in the coradical $R_{0} \in U_{q}^{0} \otimes U_{q}^{0}$ we are looking for $R$-matrices of 
the form

$$
R=R_{0} \bar{\Theta} .
$$

We remark that there is no claim that all possible $R$-matrices are of this form. However they are an interesting source of examples, motivated by the interpretation of $u_{q}(\mathfrak{g})$ as a quotient of a Drinfeld double and thus well-behaved with respect to the triangular decomposition. This ansatz has been successfully generalized to general diagonal Nichols algebras in [1]. In our more general setting $U_{q}\left(\mathfrak{g}, \Lambda, \Lambda^{\prime}\right)$, we have

$$
R_{0} \in \mathbb{C}\left[\Lambda / \Lambda^{\prime}\right] \otimes \mathbb{C}\left[\Lambda / \Lambda^{\prime}\right] .
$$

This ansatz has been worked out by Müller in his dissertation $[14,15,16]$ for small quantum groups $u_{q}(\mathfrak{g})$ which we will use in the following, leading to a system of quadratic equation on $R_{0}$ that are equivalent to $R$ being an $R$-matrix:

Theorem 2.1 (cf. [16, Theorem 8.2]). Let $u:=u_{q}(\mathfrak{g})$.

(a) There is a unique family of elements $\Theta_{\beta} \in u_{\beta}^{-} \otimes u_{\beta}^{+}, \beta \in \Lambda_{R}$, such that $\Theta_{0}=1 \otimes 1$ and $\Theta=\sum_{\beta} \Theta_{\beta} \in u \otimes u$ satisfies $\Delta(x) \Theta=\Theta \bar{\Delta}(x)$ for all $x \in u$.

(b) Let $B$ be a vector space-basis of $u^{-}$, such that $B_{\beta}=B \cap u_{\beta}^{-}$is a basis of $u_{\beta}^{-}$for all $\beta$.

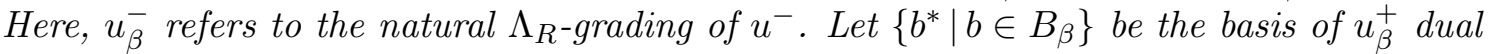
to $B_{\beta}$ under the non-degenerate bilinear form $(\cdot, \cdot): u^{-} \otimes u^{+} \rightarrow \mathbb{C}$. We have

$$
\Theta_{\beta}=(-1)^{\operatorname{tr} \beta} q_{\beta} \sum_{b \in B_{\beta}} b^{-} \otimes b^{*+} \in u_{\beta}^{-} \otimes u_{\beta}^{+} .
$$

Theorem 2.2 (cf. [16, Theorem 8.11]). Let $\Lambda^{\prime} \subset\left\{\mu \in \Lambda \mid K_{\mu}\right.$ central in $\left.u_{q}(\mathfrak{g}, \Lambda)\right\}$ a subgroup of $\Lambda$, and $G_{1}, G_{2}$ subgroups of $G:=\Lambda / \Lambda^{\prime}$, containing $\Lambda_{R} / \Lambda^{\prime}$. In the following, $\mu, \mu_{1}, \mu_{2} \in G_{1}$ and $\nu, \nu_{1}, \nu_{2} \in G_{2}$.

The element $R=R_{0} \bar{\Theta}$ with an arbitrary $R_{0}=\sum_{\mu, \nu} f(\mu, \nu) K_{\mu} \otimes K_{\nu}$ is a $R$-matrix for $u_{q}\left(\mathfrak{g}, \Lambda, \Lambda^{\prime}\right)$, if and only if for all $\alpha \in \Lambda_{R}$ and $\mu, \nu$ the following holds:

$$
\begin{aligned}
& f(\mu+\alpha, \nu)=q^{-(\nu, \alpha)} f(\mu, \nu), \quad f(\mu, \nu+\alpha)=q^{-(\mu, \alpha)} f(\mu, \nu), \\
& \sum_{\nu_{1}+\nu_{2}=\nu} f\left(\mu_{1}, \nu_{1}\right) f\left(\mu_{2}, \nu_{2}\right)=\delta_{\mu_{1}, \mu_{2}} f\left(\mu_{1}, \nu\right), \quad \sum_{\mu_{1}+\mu_{2}=\mu} f\left(\mu_{1}, \nu_{1}\right) f\left(\mu_{2}, \nu_{2}\right)=\delta_{\nu_{1}, \nu_{2}} f\left(\mu, \nu_{1}\right), \\
& \sum_{\mu} f(\mu, \nu)=\delta_{\nu, 0}, \quad \sum_{\nu} f(\mu, \nu)=\delta_{\mu, 0} .
\end{aligned}
$$

\section{Conditions for the existence of $\boldsymbol{R}$-matrices}

\subsection{A first set of conditions on $\Lambda / \Lambda^{\prime}$}

The target of our efforts is a Hopf algebra called small quantum group $u_{q}\left(\mathfrak{g}, \Lambda, \Lambda^{\prime}\right)$ with Cartan part $u_{q}^{0}=\mathbb{C}\left[\Lambda / \Lambda^{\prime}\right]$. It is defined, e.g., in [11] and depends on lattices $\Lambda, \Lambda^{\prime}$ defined below. For $\Lambda=\Lambda_{R}$ the root lattice and this is the usual small quantum group; the choice of $\Lambda^{\prime}$ differs in literature.

In the previous section we have discussed an $R=R_{0} \bar{\Theta}$-matrix for the quantum group $u_{q}\left(\mathfrak{g}, \Lambda, \Lambda^{\prime}\right)$ can be obtained from an $R_{0}$-matrix of the form

$$
R_{0}=\sum_{\mu, \nu \in \Lambda} f(\mu, \nu) K_{\mu} \otimes K_{\nu} \in \mathbb{C}\left[\Lambda / \Lambda^{\prime}\right] \otimes \mathbb{C}\left[\Lambda / \Lambda^{\prime}\right] .
$$

In the following we collect necessary and sufficient conditions for $R=R_{0} \bar{\Theta}$ to be an $R$-matrix. 
Definition 3.1. We fix once-and-for-all a finite-dimensional simple complex Lie algebra $\mathfrak{g}$ and a lattice $\Lambda$ between root- and weight-lattice

$$
\Lambda_{R} \subseteq \Lambda \subseteq \Lambda_{W}
$$

These choices have a nice geometric interpretation as quantum groups associated to different Lie groups associated to the Lie algebra $\mathfrak{g}$.

Another interesting choice is $\Lambda_{R} \subseteq \Lambda \subseteq \Lambda_{W}^{\vee} \cong \Lambda_{R}^{*}$, which would below pose no additional complications and may produce further interesting factorizable $R$-matrices.

Definition 3.2. We fix once-and-for-all a primitive $\ell$-th root of unity $q$. For $\Lambda_{1}, \Lambda_{2} \subseteq \Lambda_{W}^{\vee}$ we define the sublattice

$$
\operatorname{Cent}_{\Lambda_{1}}\left(\Lambda_{2}\right):=\left\{\nu \in \Lambda_{1} \mid(\nu, \mu) \in \ell \cdot \mathbb{Z} \forall \mu \in \Lambda_{2}\right\} .
$$

Informally, this is the centralizer with respect to the braiding $q^{-(\nu, \mu)}$.

Contrary to [11] we do not fix $\Lambda^{\prime}$ but we prove later Corollary 3.6 that there is a necessary choice for $\Lambda^{\prime}$. In this way, we get more solutions than in [11]. The only condition necessary to ensure that the Hopf algebra $u_{q}\left(\mathfrak{g}, \Lambda, \Lambda^{\prime}\right)$ is well-defined is $\Lambda^{\prime} \subseteq \operatorname{Cent}_{\Lambda_{R}}\left(\Lambda_{R}\right)$.

Theorem 3.3 (cf. [11, Theorem 3.4]). The $R_{0}$-matrix is necessarily of the form

$$
f(\mu, \nu)=\frac{1}{d\left|\Lambda_{R} / \Lambda^{\prime}\right|} \cdot q^{-(\mu, \nu)} g(\bar{\mu}, \bar{\nu}) \delta_{\bar{\mu} \in H_{1}} \delta_{\bar{\nu} \in H_{2}},
$$

where $H_{1}, H_{2}$ are subgroups of $H:=\Lambda / \Lambda_{R} \subseteq \pi_{1}$ with equal cardinality $\left|H_{1}\right|=\left|H_{2}\right|=$ : $d$ (not necessarily isomorphic!) and $g: H_{1} \times H_{2} \rightarrow \mathbb{C}^{\times}$is a pairing of groups.

The necessity of this form (in particular that the support of $f$ is indeed a subgroup!) amounts to a combinatorial problem of its own interest, which we solved for $\pi_{1}$ cyclic in [10] and for $\mathbb{Z}_{2} \times \mathbb{Z}_{2}$ by hand; a closed proof for all abelian groups would be interesting.

Definition 3.4. Let $g: G \times H \rightarrow \mathbb{C}^{\times}$be a finite group pairing, then the left radical is defined as

$$
\operatorname{Rad}_{L}(g):=\{\lambda \in G \mid g(\lambda, \eta)=1 \forall \eta \in H\} .
$$

Similarly, the right radical is defined as

$$
\operatorname{Rad}_{R}(g):=\{\eta \in H \mid g(\lambda, \eta)=1 \forall \lambda \in G\} .
$$

The pairing $g$ is called non-degenerate if $\operatorname{Rad}_{L}(g)=0$. If in $\operatorname{addition}_{\operatorname{Rad}_{R}}(g)=0, g$ is called perfect.

For an $R_{0}$-matrix of this form, a sufficient condition is that they fulfill the so-called diamondequations (see [11, Definition 2.7]) for each element $0 \neq \zeta \in\left(\operatorname{Cent}\left(\Lambda_{R}\right) \cap \Lambda\right) / \Lambda^{\prime}$.

However, we will now go into a different, more systematic direction that makes use of the following observation:

Lemma 3.5. An $R_{0}$-matrix of the form given in Theorem 3.3 is a solution to the equations in Theorem 2.2, and hence produces an $R$-matrix $R_{0} \bar{\Theta}$ iff the restriction to the support

$$
\hat{f}:=d\left|\Lambda_{R} / \Lambda^{\prime}\right| \cdot f: G_{1} \times G_{2} \rightarrow \mathbb{C}^{\times}
$$

is a perfect group pairing, where $G_{i}:=\Lambda_{i} / \Lambda^{\prime} \subseteq \Lambda / \Lambda^{\prime}=: G$. 
Proof. We first show that a solution with restriction to the support a nondegenerate pairing solves the equation.

The first equations are obviously fulfilled for the form assumed

$$
f(\mu+\alpha, \nu)=q^{-(\nu, \alpha)} f(\mu, \nu), \quad f(\mu, \nu+\alpha)=q^{-(\mu, \alpha)} f(\mu, \nu) .
$$

For the other equations the sums get only contributions in the support $\Lambda_{1} / \Lambda^{\prime} \times \Lambda_{2} / \Lambda^{\prime}$. The quantities $f(\mu, \nu) \cdot d\left|\Lambda_{R} / \Lambda^{\prime}\right|$ for fixed $\nu$ (or $\mu$ ) are characters on the respective support, and by the assumed non-degeneracy all $\nu \neq 0$ give rise to different nontrivial characters. Then the second and third relations follows from orthogonality of characters. Note that since $d\left|\Lambda_{R} / \Lambda^{\prime}\right|=\left|G_{1}\right|=\left|G_{2}\right|$ (equality of the latter was an assumption!) we were able to chose the right normalization.

For the other direction assume a solution of the given form to the equations. Then already the third equation shows that no $f(-, \nu)$ may be the trivial character and hence the form on the support is nondegenerate and hence perfect by $\left|G_{1}\right|=\left|G_{2}\right|$.

Corollary 3.6. A first consequence of the perfectness of $\hat{f}$ (i.e., a necessary condition for quasitriangularity) is

$$
\operatorname{Cent}_{\Lambda_{R}}\left(\Lambda_{1}\right)=\operatorname{Cent}_{\Lambda_{R}}\left(\Lambda_{2}\right)=\Lambda^{\prime}
$$

This fixes $\Lambda^{\prime}$ uniquely. Moreover in cases $\Lambda_{1} \neq \Lambda_{2}$, which can only happen for $\mathfrak{g}=D_{2 n}$, where $\pi_{1}$ is noncyclic, we get an additional constraint relating $\Lambda_{1}, \Lambda_{2}$.

In our case, the only possibility for $\Lambda_{1} \neq \Lambda_{2}$, s.t. $G_{1} \cong G_{2}$ is $\mathfrak{g}=D_{2 n}$. In this case, we have $\operatorname{Cent}_{\Lambda_{R}}\left(\Lambda_{W}\right)=\operatorname{Cent}_{\Lambda_{R}}\left(\Lambda_{R}\right)$ and thus the above condition is always fulfilled.

Our main goal for the new approach on quasitriangularity as well as the later modularity is to reduce this non-degeneracy condition for $\hat{f}$ to a non-degeneracy condition for $g$ on $H_{1}, H_{2} \subset \pi_{1}$ that can be checked explicitly.

\subsection{A natural form on the fundamental group}

We now define for each triple $\left(\Lambda, \Lambda_{1}, \Lambda_{2}\right)$ and each $\ell$ th root of unity $q$ a natural pairing $a_{\ell}$ on the subgroups $H_{i}:=\Lambda_{i} / \Lambda_{R}$ of the fundamental group $\pi_{1}:=\Lambda_{W} / \Lambda_{R}$. The simplest example is $a_{\ell}=e^{-2 \pi i(\mu, \nu)}$. In general it is a transportation of the natural form $q^{-(\mu, \nu)}$ (which does not factorize over $\Lambda_{R}$ ) to $H_{i}$ by a suitable isomorphism $A_{\ell}$.

This isomorphism $A_{\ell}$ will encapsulate the crucial dependence on the common divisors of $\ell,|H|$ and the root lengths $d_{i}$; moreover, for different $H$ these forms are not simply restrictions of one another.

Then, we can moreover transport any given pairing $g$ together with $q^{-(\mu, \nu)}$ along the isomorphism $A_{\ell}$ to the $H_{i}$ and thus define forms $a_{\ell}^{g}$ on $H$. The main result of this section is in Theorem 3.13 that the non-degeneracy condition in Lemma 3.5 for $R_{0}(f)$ depending on $H_{i}, g$ is equivalent to $a_{\ell}^{g}$ being non-degenerate.

Definition 3.7. Let $\Lambda \subseteq \Lambda_{W}^{\vee}$ be a sublattice, s.t. $\Lambda_{R} \subseteq \Lambda$. By $\hat{\Lambda} \subset \Lambda_{W}^{\vee}$ we denote the unique sublattice, s.t. the symmetric bilinear form $(\cdot, \cdot): \Lambda_{W}^{\vee} \times \Lambda_{W}^{\vee} \rightarrow \mathbb{Q}$ induces a commuting diagram

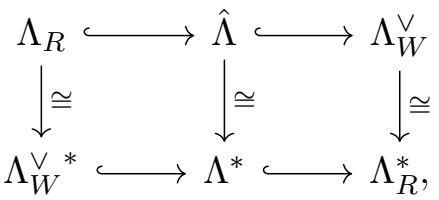

where $\Lambda^{*}:=\operatorname{Hom}_{\mathbb{Z}}(\Lambda, \mathbb{Z})$. In particular, we have $\hat{\Lambda}_{R}=\Lambda_{W}^{\vee}$ and $\hat{\Lambda}_{W}^{\vee}=\Lambda_{R}$. 
Definition 3.8. A centralizer transfer map is an group endomorphism $A_{\ell} \in \operatorname{End}_{\mathbb{Z}}(\Lambda)$, s.t.

1) $A_{\ell}(\Lambda) \stackrel{!}{=} \Lambda \cap \ell \cdot \hat{\Lambda}_{R}=\operatorname{Cent}_{\Lambda}^{\ell}\left(\Lambda_{R}\right)$,

2) $A_{\ell}\left(\Lambda_{R}\right) \stackrel{!}{=} \Lambda_{R} \cap \ell \cdot \hat{\Lambda}=\operatorname{Cent}_{\Lambda_{R}}^{\ell}(\Lambda)$.

Such a $A_{\ell}$ induces a group isomorphism

$$
\Lambda / \Lambda_{R} \stackrel{\sim}{\longrightarrow} \operatorname{Cent}_{\Lambda}^{\ell}\left(\Lambda_{R}\right) / \operatorname{Cent}_{\Lambda_{R}}^{\ell}(\Lambda) .
$$

Of course $A_{\ell}$ is not unique.

Question 3.9. Are there abstract arguments for the existence of these isomorphism and for its explicit form?

We will calculate explicit expressions for $A_{\ell}$ depending on the cases in the next section. At this point we give the generic answers:

Example 3.10. For $\Lambda=\Lambda_{W}^{\vee}$ we have $A_{\ell}=\ell \cdot$ id.

For $\Lambda=\Lambda_{R}$ the two conditions are equivalent, so existence is trivial (resp. obviously the two trivial groups are isomorphic) and we may simply take for $A_{\ell}$ any base change between left and right side. The expression may however be nontrivial.

Lemma 3.11. Assume $\operatorname{gcd}\left(\ell,\left|\Lambda_{W}^{\vee} / \Lambda\right|\right)=1$, then $A_{\ell}=\ell \cdot \mathrm{id}$. In particular this is the case if $\ell$ is prime to all root lengths and all divisors of the Cartan matrix.

Moreover if $\ell=\ell_{1} \ell_{2}$ with $\operatorname{gcd}\left(\ell_{1},\left|\Lambda_{W}^{\vee} / \Lambda\right|\right)=1$, then $A_{\ell}=\ell_{1} \cdot A_{\ell_{2}}$.

This means we only have to calculate $A_{\ell}$ for all divisors $\ell$ of $\left|\Lambda_{W}^{\vee} / \Lambda\right|$, which is a subset of all divisors of root lengths times divisors of the Cartan matrix.

Proof. For the first condition we need to show for any $\lambda \in \Lambda_{W}^{\vee}$ that $\ell \lambda \in \Lambda$ already implies $\lambda \in \Lambda$. But if by assumption the order of the quotient group $\Lambda_{W}^{\vee} / \Lambda$ is prime to $\ell$, then $\ell$. is an isomorphism on this abelian group, hence follows the assertion. For the second condition applies the same argument noting that $\left|\hat{\Lambda} / \Lambda_{R}\right|=\left|\Lambda_{W}^{\vee} / \Lambda\right|$.

For the second claim we simply consider the inclusion chains

$$
\begin{aligned}
& A_{\ell}(\Lambda) \subset \Lambda \cap \ell_{2} \cdot \hat{\Lambda}_{R} \subset \Lambda \cap \ell \cdot \hat{\Lambda}_{R}, \\
& A_{\ell}\left(\Lambda_{R}\right) \subset \Lambda \cap \ell_{2} \cdot \hat{\Lambda} \subset \Lambda_{R} \cap \ell \cdot \hat{\Lambda},
\end{aligned}
$$

where a first isomorphism is given by $A_{\ell_{2}}$ and again $\ell_{1}$. is a second isomorphism because it is prime to the index.

Our main result of this chapter is the following:

Theorem 3.12. Let $\Lambda_{R} \subseteq \Lambda_{1}, \Lambda_{2} \subseteq \Lambda_{W}$ be intermediate lattices, s.t. the condition in Corollary 3.6 is fulfilled, i.e., $\operatorname{Cent}_{\Lambda_{R}}\left(\Lambda_{1}\right)=\operatorname{Cent}_{\Lambda_{R}}\left(\Lambda_{2}\right)=\Lambda^{\prime}$. Assume we have a centralizer transfer map $A_{\ell}$.

1. The following form is well defined on the quotients:

$$
\begin{aligned}
a_{g}^{\ell}: & \Lambda_{1} / \Lambda_{R} \times \Lambda_{2} / \Lambda_{R} \longrightarrow \mathbb{C}^{\times}, \\
& (\bar{\lambda}, \bar{\mu}) \longmapsto q^{-\left(\lambda, A_{\ell}(\mu)\right)} \cdot g\left(\lambda, A_{\ell}(\mu)\right) .
\end{aligned}
$$

2. Let

$$
\operatorname{Cent}_{\Lambda_{1}}^{g}\left(\Lambda_{2}\right):=\left\{\lambda \in \Lambda_{1} \mid q^{(\lambda, \mu)}=g(\lambda, \mu) \forall \mu \in \Lambda_{2}\right\} .
$$

Then the inclusion $\operatorname{Cent}_{\Lambda_{1}}^{g}\left(\Lambda_{2}\right) \hookrightarrow \Lambda_{1}$ induces an isomorphism

$$
\operatorname{Cent}_{\Lambda_{1}}^{g}\left(\Lambda_{2}\right) / \Lambda^{\prime} \cong \operatorname{Rad}\left(a_{g}^{\ell}\right)
$$


Corollary 3.13. The quasitriangularity conditions for a choice $R_{0}$ are by Lemma 3.5 equivalent to the non-degeneracy of the group pairing on $\Lambda_{1} / \Lambda^{\prime} \times \Lambda_{2} / \Lambda^{\prime}$ :

$$
\hat{f}(\lambda, \mu)=q^{-(\lambda, \mu)} g(\lambda, \mu) .
$$

By the previous theorem this condition is now equivalent to the nondegeneracy of $a_{g}^{\ell}$.

This condition on the fundamental group, which is a finite abelian group and mostly cyclic, can be checked explicitly once $a_{g}^{\ell}$ has been calculated.

Proof of Theorem 3.12. The first part of the theorem is a direct consequence of the definition of the centralizer transfer matrix $A_{\ell}$. For the second part, we first notice that by assumption we have a commutative diagram of finite abelian groups

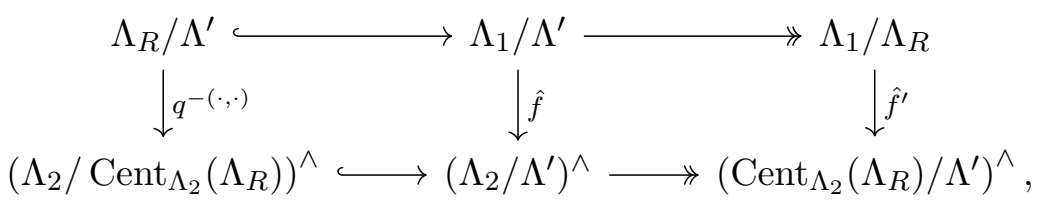

where $G^{\wedge}$ denotes the dual group of a group $G$.

Now, by the five lemma we know that $\hat{f}$ is an isomorphism if and only if the induced map $\hat{f}^{\prime}$ is an isomorphism. Post-composing this map with the dualized centralizer transfer matrix $A_{\ell}^{\wedge}:\left(\operatorname{Cent}_{\Lambda_{2}}\left(\Lambda_{R}\right) / \Lambda^{\prime}\right)^{\wedge} \cong\left(\Lambda_{2} / \Lambda_{R}\right)^{\wedge}$ gives $a_{g}^{\ell}$.

\section{Explicit calculation for every $\mathfrak{g}$}

In the following, we want to compute the endomorphism $A_{\ell} \in \operatorname{End}_{\mathbb{Z}}(\Lambda)$ and the pairing $a_{\ell}$ on the fundamental group explicitly in terms of the Cartan matrices and the common divisors of $\ell$ with root lengths and divisors of the Cartan matrix. We will finally give a list for all $\mathfrak{g}$.

\subsection{Technical tools}

We choose the basis of simple roots $\alpha_{i}$ for $\Lambda_{R}$ and the dual basis of fundamental coweights $\lambda_{i}^{\vee}$ for the dual lattice $\Lambda_{W}^{\vee}$ with $\left(\alpha_{i}, \lambda_{j}^{\vee}\right)=\delta_{i, j}$.

For any choice $\Lambda \subset \Lambda_{W} \subset \Lambda_{W}^{\vee}$, let $A_{\Lambda}$ be a basis matrix, i.e., any $\mathbb{Z}$-linear isomorphism $\Lambda_{W}^{\vee} \rightarrow \Lambda$ sending the basis $\lambda_{i}^{\vee}$ of $\Lambda_{W}^{\vee}$ to some basis $\mu_{i}$ of $\Lambda$. It is unique up to pre-composition of a unimodular matrix $U \in \mathrm{SL}_{n}(\mathbb{Z})$.

The dual basis $A_{\hat{\Lambda}}$ of $\hat{\Lambda}$ is defined by

$$
\left(A_{\hat{\Lambda}}\left(\lambda_{i}^{\vee}\right), A_{\Lambda}\left(\lambda_{j}^{\vee}\right)\right)=\delta_{i j}
$$

Explicitly, $A_{\hat{\Lambda}}$ is given by $A_{\hat{\Lambda}}=\left(A_{\Lambda}^{-1} A_{R}\right)^{T}$, where $\left(A_{R}\right)_{i j}=\left(\alpha_{i}, \alpha_{j}\right)$. Now, let $A_{\Lambda}=P_{\Lambda} S_{\Lambda} Q_{\Lambda}$ be the unique Smith decomposition of $A_{\Lambda}$, which means: $P_{\Lambda}, Q_{\Lambda}$ are unimodular and $S_{\Lambda}$ is diagonal with diagonal entries $\left(S_{\Lambda}\right)_{i i}=: d_{i}^{\Lambda}$, such that $d_{i}^{\Lambda} \mid d_{j}^{\Lambda}$ for $i<j$.

Example 4.1. For the root lattice the $d_{i}^{\Lambda_{R}}$ are the divisors of scalar product matrix $\left(\alpha_{i}, \alpha_{j}\right)$. Their product is

$$
\prod_{i} d_{i}^{\Lambda_{R}}=\left|\Lambda_{W}^{\vee} / \Lambda_{R}\right|=\left(\prod_{i} d_{i}\right) \cdot\left|\pi_{1}\right|, \quad d_{i}=\frac{\left(\alpha_{i}, \alpha_{i}\right)}{2} .
$$

For the coweight lattice all $d_{i}^{\Lambda_{W}^{\vee}}=1$. For the weight lattice we recover the familiar $d_{i}^{\Lambda_{W}}=d_{i}$. 
Without loss of generality, we will assume the basis matrices $A_{\Lambda}$ to be symmetric, i.e., $Q_{\Lambda}=P_{\Lambda}^{T}$. We then have the following lemma:

Lemma 4.2. Let $\Lambda_{R} \subseteq \Lambda \subseteq \Lambda_{W}^{\vee}$ be a lattice. We define lattices

$$
A_{\text {Cent }}:=\left(P_{\Lambda}^{T}\right)^{-1} D_{\ell} P_{\Lambda}^{-1}, \quad D_{\ell}:=\operatorname{Diag}\left(\frac{\ell}{\operatorname{gcd}\left(\ell, d_{i}^{\Lambda}\right)}\right) .
$$

Then,

$$
\operatorname{Cent}_{\Lambda_{R}}(\Lambda)=A_{R} A_{\text {Cent }} \Lambda_{W}^{\vee}, \quad \operatorname{Cent}_{\Lambda}\left(\Lambda_{R}\right)=A_{\Lambda} A_{\text {Cent }} \Lambda_{W}^{\vee}
$$

Proof. We compute explicitly,

$$
\begin{aligned}
\operatorname{Cent}_{\Lambda_{R}}(\Lambda) & =\Lambda_{R} \cap \ell \cdot \hat{\Lambda}=A_{R} \Lambda_{W}^{\vee} \cap\left(A_{\Lambda}^{-1} A_{R}\right)^{T} \ell \Lambda_{W}^{\vee} \\
& =\left(A_{\Lambda}^{-1} A_{R}\right)^{T}\left(\left(\left(A_{\Lambda}^{-1} A_{R}\right)^{T}\right)^{-1} A_{R} \Lambda_{W}^{\vee} \cap \ell \Lambda_{W}^{\vee}\right)=A_{R} A_{\Lambda}^{-1}\left(A_{\Lambda} \cap \ell \Lambda_{W}^{\vee}\right) \\
& =A_{R}\left(P_{\Lambda} S_{\Lambda} P_{\Lambda}^{T}\right)^{-1}\left(P_{\Lambda} S_{\Lambda} P_{\Lambda}^{T} \Lambda_{W}^{\vee} \cap \ell \Lambda_{W}^{\vee}\right)=A_{R}\left(P_{\Lambda}^{T}\right)^{-1} S_{\Lambda}^{-1}\left(S_{\Lambda} \Lambda_{W}^{\vee} \cap \ell \Lambda_{W}^{\vee}\right) \\
& =A_{R}\left(P_{\Lambda}^{T}\right)^{-1} S_{\Lambda}^{-1} \operatorname{Diag}\left(\operatorname{lcm}\left(S_{\Lambda_{i i}}, \ell\right)\right) \Lambda_{W}^{\vee}=A_{R}\left(P_{\Lambda}^{T}\right)^{-1} D_{\ell} \Lambda_{W}^{\vee}=A_{R} A_{\text {Cent }} \Lambda_{W}^{\vee} .
\end{aligned}
$$

On the other hand,

$$
\begin{aligned}
\operatorname{Cent}_{\Lambda}\left(\Lambda_{R}\right) & =\Lambda \cup \ell \hat{\Lambda}_{R}=\Lambda \cup \ell \Lambda_{W}^{\vee}=A_{\Lambda} \Lambda_{W}^{\vee} \cup \ell \Lambda_{W}^{\vee}=P_{\Lambda} S_{\Lambda} P_{\Lambda}^{T} \Lambda_{W}^{\vee} \cup \ell \Lambda_{W}^{\vee} \\
& =P_{\Lambda}\left(S_{\Lambda} \Lambda_{W}^{\vee} \cup \ell \Lambda_{W}^{\vee}\right)=P_{\Lambda} S_{\Lambda} D_{\ell} \Lambda_{W}^{\vee}=A_{\Lambda}\left(P_{\Lambda}^{T}\right)^{-1} D_{\ell} \Lambda_{W}^{\vee}=A_{\Lambda} A_{\text {Cent }} \Lambda_{W}^{\vee} .
\end{aligned}
$$

In particular, this means $A_{\hat{\Lambda}} \operatorname{Cent}_{\Lambda}\left(\Lambda_{R}\right)=\operatorname{Cent}_{\Lambda_{R}}(\Lambda)$.

\subsection{Case $\Lambda=\Lambda_{W}$}

In order to exhaust all cases that appear in our setting, we continue with $\Lambda=\Lambda_{W}$ :

Lemma 4.3. In the case $\Lambda=\Lambda_{W}$, the centralizer transfer matrix $A_{\ell}$ is of the following form:

$$
A_{\ell}= \begin{cases}A_{\Lambda_{W}} A_{\mathrm{Cent}} Q_{C}^{T} P_{C}^{-1} A_{\Lambda_{W}}^{-1}, & \operatorname{gcd}\left(\ell,\left|\pi_{1}\right|\right) \neq 1, \\ \ell \cdot \mathrm{id}, & \text { else. }\end{cases}
$$

Here, $C=P_{C} S_{C} Q_{C}$ denotes the Smith decomposition of the Cartan matrix of $\mathfrak{g}$.

Proof. As we noted in Example 4.1, we have $A_{\Lambda_{W}}=\operatorname{Diag}\left(d_{i}\right)$, for $d_{i}$ being the $i$ th root length. Since $d_{i} \in\{1, p\}$ for some prime number $p$, up to a permutation $A_{\Lambda_{W}}$ is already in Smith normal form: this means that $P_{\Lambda_{W}}$ is a permutation matrix of the form $\left(P_{\Lambda_{W}}\right)_{i j}=\delta_{j, \sigma(i)}$ for some $\sigma \in S_{n}$, s.t. $d_{\sigma(1)} \leq \cdots \leq d_{\sigma(n)}$. It follows that $A_{\text {Cent }}=\operatorname{Diag}\left(\frac{\ell}{\operatorname{gcd}\left(\ell, d_{i}\right)}\right)$.

Using the definition $C_{i j}=\frac{\left(\alpha_{i}, \alpha_{j}\right)}{d_{i}}$, in the case $\operatorname{gcd}\left(\ell,\left|\pi_{1}\right|\right) \neq 1$ we obtain

$$
A_{\text {Cent }} C^{T}=C A_{\text {Cent }} \text {. }
$$

Thus,

$$
\begin{aligned}
A_{\ell} A_{R} & =A_{\Lambda_{W}} A_{\mathrm{Cent}} Q_{C}^{T} P_{C}^{-1} A_{\Lambda_{W}}^{-1} A_{R}=A_{R} C^{-1} A_{\mathrm{Cent}} Q_{C}^{T} P_{C}^{-1} C \\
& =A_{R} A_{\text {Cent }}\left(C^{T}\right)^{-1} Q_{C}^{T} P_{C}^{-1} C=A_{R} A_{\text {Cent }} .
\end{aligned}
$$

By the previous lemma, this proves the first condition for $A_{\ell}$. The second condition follows immediately from the previous lemma.

The case $\operatorname{gcd}\left(\ell,\left|\pi_{1}\right|\right)=1$ follows from Lemma 3.11 and the fact that $\left|\pi_{1}\right|=\left|\Lambda_{W}^{\vee} / \Lambda_{R}^{\vee}\right|$. 


\subsection{Case $A_{n}$}

In the following example, we treat the case $\mathfrak{g}=A_{n}$ with fundamental group $\Lambda_{W} / \Lambda_{R}=\mathbb{Z}_{n+1}$ for general intermediate lattices $\Lambda_{R} \subseteq \Lambda \subseteq \Lambda_{W}$.

Example 4.4. In order to compute the centralizer transfer map $A_{\ell}$, we first compute the Smith decomposition of $A_{R}$ :

$$
\begin{aligned}
& A_{R}=\left(\begin{array}{cccccc}
2 & -1 & 0 & \ldots & & 0 \\
-1 & 2 & -1 & 0 & & 0 \\
0 & -1 & 2 & \ddots & \ddots & \vdots \\
0 & 0 & \ddots & \ddots & -1 & 0 \\
\vdots & & \ddots & -1 & 2 & -1 \\
0 & \ldots & & 0 & -1 & 2
\end{array}\right)
\end{aligned}
$$

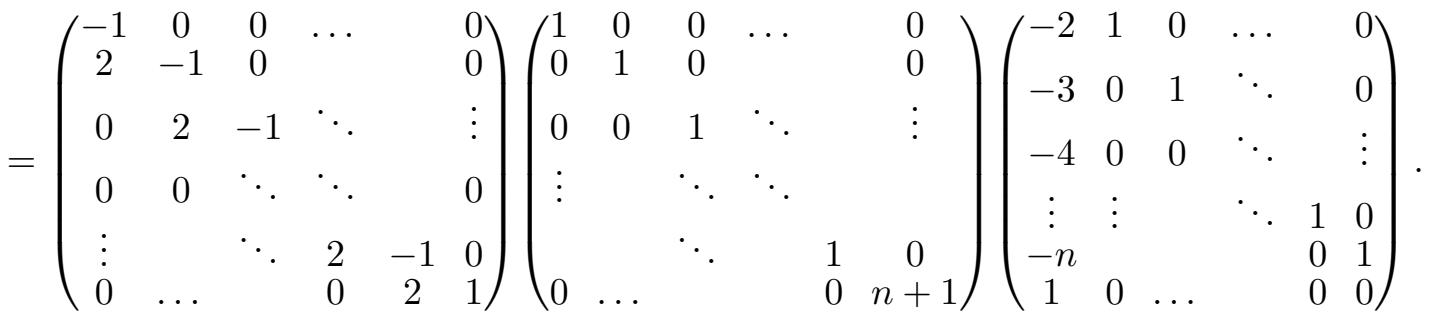

A sublattice $\Lambda_{R} \subsetneq \Lambda \subsetneq \Lambda_{W}$ is uniquely determined by a divisor $d \mid n+1$, so that $\Lambda / \Lambda_{R} \cong \mathbb{Z}_{d}$ and is generated by the multiple $\hat{d} \lambda_{n}$, where $\hat{d}:=\frac{n+1}{d}$. Then

$$
d_{i}^{\Lambda}= \begin{cases}1, & i<n, \\ d, & i=n\end{cases}
$$

Since $A_{n}$ is simply laced with cyclic fundamental group, the formula $A_{\Lambda}=P_{R} S_{\Lambda} P_{R}^{T}$ gives us symmetric basis matrices of sublattices $\Lambda_{R} \subseteq \Lambda \subseteq \Lambda_{W}$. We also substitute the above basis matrix of the root lattice $A_{R}$ by $A_{R}\left(Q_{R}\right)^{-1} P_{R}^{T}$. It is then easy to see that the definition $A_{\ell}:=P_{R} D_{\ell} P_{R}^{T}$ gives a centralizer transfer matrix. We calculate it explicitly

$$
\left(A_{\ell}\right)_{i j}=\left(P_{R} D_{\ell} P_{R}^{-1}\right)_{i j}= \begin{cases}\delta_{i j}, & i<n, \\ (n+1-j)\left(\frac{\ell}{\operatorname{gcd}(\ell, d)}-1\right), & i=n \text { and } j<n, \\ \frac{\ell}{\operatorname{gcd}(\ell, d)}, & i=j=n .\end{cases}
$$

Now a form $g$ is uniquely determined by a $d$ th root of unity $g(\chi, \chi)=\exp \left(\frac{2 \pi i \cdot k}{d}\right)=\zeta_{d}^{k}$ with some $k$. Then we calculate the form $a_{g}^{\ell}$ on the generator

$$
\begin{aligned}
a_{g}^{\ell}(\chi, \chi) & =q^{-\left(\chi, A_{\ell}(\chi)\right)} g\left(\chi, A_{\ell}(\chi)\right)=q^{-\frac{(n+1)^{2} \cdot \ell}{d^{2} \operatorname{gcd}(\ell, \hat{d})}\left(\lambda_{n}^{\vee}, \lambda_{n}^{\vee}\right)} \cdot g(\chi, \chi)^{\frac{\ell}{\operatorname{gcd}(\ell, \hat{d})}} \\
& =\exp \left(\frac{2 \pi i \cdot(k \ell-\hat{d} n)}{d \cdot \operatorname{gcd}(\ell, \hat{d})}\right) .
\end{aligned}
$$

For example the trivial $g$ (i.e., $k=0$ ) gives an $R$-matrix for all lattices $\Lambda$ (defined by $\hat{d} d=n+1$ ) iff $\frac{\hat{d}}{\operatorname{gcd}(\ell, \hat{d})}$ is coprime to $d$. For $\ell$ coprime to the divisor $n+1$ this amounts to all lattices associated to decompositions of $n+1$ into two coprime factors. 


\subsection{Case $D_{n}$}

Finally, we consider the root lattice $D_{n}$. Since we have $\pi_{1}\left(D_{2 n \geq 4}\right) \cong \mathbb{Z}_{2} \times \mathbb{Z}_{2}$ and $\pi_{1}\left(D_{2 n+1 \geq 5}\right) \cong$ $\mathbb{Z}_{4}$, it is appropriate to split this investigation in two steps. We start with $D_{2 n \geq 4}$. In order to compute the respective Smith decompositions, we used the software Wolfram Mathematica.

Example 4.5. In the case $D_{2 n \geq 4}$, we have three different possibilities for the lattices $\Lambda_{R} \subseteq$ $\Lambda_{1}, \Lambda_{2} \subseteq \Lambda_{W}$ :

1. $\Lambda_{1} \neq \Lambda_{2}, H_{1} \cong H_{2} \cong \mathbb{Z}_{2}$ : In this case, the subgroups $\Lambda_{i} / \Lambda_{R} \subseteq \Lambda_{R}$ are spanned by the fundamental weights $\lambda_{2(n-1)+i}$. As in the case $A_{n}$, we define the centralizer transfer map $A_{\ell}:=P_{R} D_{\ell} P_{R}^{-1}$ on $H_{2}$. This is possible since the symmetric basis matrix $A_{\Lambda_{2}}=P_{R} S_{\Lambda_{2}} P_{R}^{T}$ of $\Lambda_{2}$ is already in Smith normal form. Using the software Wolfram Mathematica in order to compute $P_{R}$, we obtain $A_{\ell}\left(\lambda_{2 n}\right)=\frac{\ell}{\operatorname{gcd}(2, \ell)}$. Combining this with $\left(\lambda_{2 n-1}, \lambda_{2 n}\right)=\frac{n-1}{2}$, we get

$$
a_{g}^{\ell}\left(\lambda_{2 n-1}, \lambda_{2 n}\right)=\exp \left(\frac{2 \pi i \cdot(k l-2(n-1))}{2 \cdot \operatorname{gcd}(2, \ell)}\right)
$$

for $g\left(\lambda_{2 n-1}, \lambda_{2 n}\right)=\exp \left(\frac{2 \pi i k}{2}\right)$.

2. $\Lambda_{1}=\Lambda_{2}, H_{i} \cong \mathbb{Z}_{2}$ : Without restrictions and in order to use the same definition for $A_{\ell}$ as above, we choose $\Lambda_{i}$, s.t. the group $\Lambda_{i} / \Lambda_{R}$ is spanned by $\lambda_{2 n}$. Combining the above result $A_{\ell}\left(\lambda_{2 n}\right)=\frac{\ell}{\operatorname{gcd}(2, \ell)}$ with $\left(\lambda_{2 n}, \lambda_{2 n}\right)=\frac{n}{2}$, we obtain

$$
a_{g}^{\ell}\left(\lambda_{2 n}, \lambda_{2 n}\right)=\exp \left(\frac{2 \pi i \cdot(k l-2 n)}{2 \cdot \operatorname{gcd}(2, \ell)}\right)
$$

for $g\left(\lambda_{2 n}, \lambda_{2 n}\right)=\exp \left(\frac{2 \pi i k}{2}\right)$.

3. $\Lambda_{1}=\Lambda_{2}=\Lambda_{W}, H \cong \mathbb{Z}_{2} \times \mathbb{Z}_{2}$ : A group pairing $g:\left(\mathbb{Z}_{2} \times \mathbb{Z}_{2}\right) \times\left(\mathbb{Z}_{2} \times \mathbb{Z}_{2}\right) \rightarrow \mathbb{C}^{\times}$is uniquely defined by a matrix $K \in \mathfrak{g l}\left(2, \mathbb{F}_{2}\right)$, so that

$$
g\left(\lambda_{2(n-1)+i}, \lambda_{2(n-1)+j}\right)=\exp \left(\frac{2 \pi i K_{i j}}{2}\right) .
$$

Since $D_{n}$ is simply-laced, we have $A_{\ell}=\ell \cdot$ id. Using $\left(\lambda_{2(n-1)+i}, \lambda_{2(n-1)+j}\right) \bmod 2=\delta_{i+j \text { odd }}$, we obtain

$$
a_{\ell}^{g}\left(\lambda_{2(n-1)+i}, \lambda_{2(n-1)+j}\right)=\exp \left(\frac{2 \pi i \cdot K_{i j} \ell}{2}\right)(-1)^{i+j} .
$$

The last step is the case $D_{2 n+1 \geq 5}$ :

Example 4.6. Since it it is simply-laced and its fundamental group is cyclic, the case $D_{2 n+1 \geq 5}$ can be treated very similar to $A_{n}$. We distinguish two cases:

1. $\Lambda_{1}=\Lambda_{2}, H_{i}=\left\langle 2 \lambda_{2 n+1}\right\rangle \cong \mathbb{Z}_{2}$. As in the case $A_{n}$, we define the centralizer transfer map $A_{\ell}:=P_{R} D_{\ell} P_{R}^{-1}$ on $H_{2}$. Using $\left(\lambda_{2 n+1}, \lambda_{2 n+1}\right)=\frac{2 n+1}{4}$, we obtain

$$
a_{g}^{\ell}\left(2 \lambda_{2 n+1}, 2 \lambda_{2 n+1}\right)=\exp \left(\frac{2 \pi i \cdot(k \ell-2(2 n+1))}{2 \cdot \operatorname{gcd}(2, \ell)}\right) .
$$

for $g\left(2 \lambda_{2 n+1}, 2 \lambda_{2 n+1}\right)=\exp \left(\frac{2 \pi i k}{2}\right)$.

2. $\Lambda_{1}=\Lambda_{2}=\Lambda_{W}, H=\left\langle\lambda_{2 n+1}\right\rangle \cong \mathbb{Z}_{4}$. By an analogous argument as above, we obtain

$$
a_{g}^{\ell}\left(\lambda_{2 n+1}, \lambda_{2 n+1}\right)=\exp \left(\frac{2 \pi i \cdot(k \ell-(2 n+1))}{4}\right)
$$

for $g\left(\lambda_{2 n+1}, \lambda_{2 n+1}\right)=\exp \left(\frac{2 \pi i k}{4}\right)$. 


\subsection{Table of all quasitriangular quantum groups}

In the following table, we list all simple Lie algebras and check for which non-trivial choices of $\Lambda, \Lambda_{i}, \ell$ and $g$ the element $R_{0} \bar{\Theta}$ is an $R$-matrix. As before, we define $H_{i}:=\Lambda_{i} / \Lambda_{R}$ and $H:=\Lambda / \Lambda_{R}$. In the cyclic case, if $x_{i}$ are generators of the $H_{i}$, then the pairing is uniquely defined by an element $1 \leq k \leq\left|H_{i}\right|$, s.t. $g\left(x_{1}, x_{2}\right)=\exp \left(\frac{2 \pi i k}{\left|H_{i}\right|}\right)$. In the case $D_{2 n}, \Lambda=\Lambda_{W}, g$ is uniquely defined by a $2 \times 2$-matrix $K \in \mathfrak{g l}\left(2, \mathbb{F}_{2}\right)$, s.t. $g\left(\lambda_{2(n-1)+i}, \lambda_{2(n-1)+j}\right)=\exp \left(\frac{2 \pi i K_{i j}^{g}}{2}\right)$ for $i, j \in\{1,2\}$.

The columns of the following table are labeled by

1) the finite-dimensional simple complex Lie algebra $\mathfrak{g}$,

2) the natural number $\ell$, determining the root of unity $q=\exp \left(\frac{2 \pi i}{\ell}\right)$,

3 ) the number of possible $R$-matrices for the Lusztig ansatz,

4) the subgroups $H_{i} \subseteq H=\Lambda / \Lambda_{R}$ introduced in Theorem 3.3,

5) the subgroups $H_{i}$ in terms of generators given by multiples of fundamental dominant weights $\lambda_{i} \in \Lambda_{W}$,

6) the group pairing $g: H_{1} \times H_{2} \rightarrow \mathbb{C}^{\times}$determined by its values on generators,

7) the group pairing $a_{g}^{\ell} \subseteq \Lambda / \Lambda^{\prime}$ introduced in Theorem 3.12 determined by its values on generators.

\begin{tabular}{|c|c|c|c|c|c|c|}
\hline $\mathfrak{g}$ & $\ell$ & \# & $H_{i} \cong$ & $H_{i(i=1,2)}$ & $g$ & $a_{g}^{\ell}$ \\
\hline all & & 1 & $\mathbb{Z}_{1}$ & $\langle 0\rangle$ & $g=1$ & 1 \\
\hline $\begin{array}{c}A_{n \geq 1} \\
\pi_{1}=\mathbb{Z}_{n+1}\end{array}$ & & $\infty$ & $\left|\begin{array}{c}\mathbb{Z}_{d} \\
d \mid n+1\end{array}\right|$ & $\begin{array}{c}\left\langle\hat{d} \lambda_{n}\right\rangle \\
\hat{d}=\frac{n+1}{d}\end{array}$ & $\begin{array}{c}g\left(\hat{d} \lambda_{n}, \hat{d} \lambda_{n}\right)=\exp \left(\frac{2 \pi i k}{d}\right) \\
\operatorname{gcd}\left(d, \frac{k \ell-\hat{d} n}{\operatorname{gcd}(\ell, \hat{d})}\right)=1\end{array}$ & $\exp \left(\frac{2 \pi i \cdot(k \ell-\hat{d} n)}{d \cdot \operatorname{gcd}(\ell, \hat{d})}\right)$ \\
\hline \multirow{2}{*}{$\begin{array}{c}B_{n \geq 2} \\
\pi_{1}=\mathbb{Z}_{2}\end{array}$} & $\ell$ even & 2 & \multirow{2}{*}{$\mathbb{Z}_{2}$} & \multirow{2}{*}{$\left\langle\lambda_{n}\right\rangle$} & $g\left(\lambda_{n}, \lambda_{n}\right)= \pm 1$ & -1 \\
\hline & $\ell$ odd & 1 & & & $g\left(\lambda_{n}, \lambda_{n}\right)=(-1)^{n+1}$ & $\exp \left(\frac{2 \pi i \cdot(k \ell-n)}{2}\right)$ \\
\hline \multirow{3}{*}{$\begin{array}{c}C_{n \geq 3} \\
\pi_{1}=\mathbb{Z}_{2}\end{array}$} & $\ell \equiv 2 \bmod 4$ & 1 & \multirow{3}{*}{$\mathbb{Z}_{2}$} & \multirow{3}{*}{$\left\langle\lambda_{n}\right\rangle$} & $g\left(\lambda_{n}, \lambda_{n}\right)=1$ & $\exp \left(\frac{2 \pi i \cdot\left(k \frac{\ell}{2}+1\right)}{2}\right)$ \\
\hline & $\ell \equiv 0 \bmod 4$ & 2 & & & $g\left(\lambda_{n}, \lambda_{n}\right)= \pm 1$ & -1 \\
\hline & $\ell$ odd & 1 & & & $g\left(\lambda_{n}, \lambda_{n}\right)=-1$ & $\exp \left(\frac{2 \pi i \cdot(k \ell-2 n)}{2}\right)$ \\
\hline \multirow{8}{*}{$\begin{array}{c}D_{2 n \geq 4} \\
\pi_{1}=\mathbb{Z}_{2} \times \mathbb{Z}_{2}\end{array}$} & $\ell \equiv 2 \bmod 4$ & 1 & \multirow{3}{*}{$\mathbb{Z}_{2}$} & \multirow{3}{*}{$\begin{array}{c}H_{1} \cong\left\langle\lambda_{2 n-1}\right\rangle \\
H_{2} \cong\left\langle\lambda_{2 n}\right\rangle\end{array}$} & $g\left(\lambda_{2 n-1}, \lambda_{2 n}\right)=(-1)^{n}$ & \multirow{2}{*}{$\exp \left(\frac{\left.2 \pi i \cdot\left(k \frac{\ell}{2}-n+1\right)\right)}{2}\right)$} \\
\hline & $\ell \equiv 0 \bmod 4$ & $2 \delta_{2 \mid n}$ & & & $g\left(\lambda_{2 n-1}, \lambda_{2 n}\right)= \pm 1, n$ even & \\
\hline & $\ell$ odd & 1 & & & $g\left(\lambda_{2 n-1}, \lambda_{2 n}\right)=-1$ & $\exp \left(\frac{2 \pi i \cdot(k \ell-2(n-1))}{2}\right)$ \\
\hline & $\ell \equiv 2 \bmod 4$ & 1 & \multirow{3}{*}{$\mathbb{Z}_{2}$} & \multirow{3}{*}{$\left\langle\lambda_{2 n}\right\rangle$} & $g\left(\lambda_{2 n}, \lambda_{2 n}\right)=(-1)^{n+1}$ & \multirow{2}{*}{$\exp \left(\frac{2 \pi i\left(k \frac{\ell}{2}-n\right)}{2}\right)$} \\
\hline & $\ell \equiv 0 \bmod 4$ & $2 \delta_{2 \nmid n}$ & & & $g\left(\lambda_{2 n}, \lambda_{2 n}\right)= \pm 1, n$ odd & \\
\hline & $\ell$ odd & 1 & & & $g\left(\lambda_{2 n}, \lambda_{2 n}\right)=-1$ & $\exp \left(\frac{2 \pi i(k \ell-2 n)}{2}\right)$ \\
\hline & $\ell$ even & 16 & \multirow{2}{*}{$\mathbb{Z}_{2} \times \mathbb{Z}_{2}$} & \multirow{2}{*}{$\left\langle\lambda_{2 n}, \lambda_{2 n+1}\right\rangle$} & $g\left(\lambda_{2(n-1)+i}, \lambda_{2(n-1)+j}\right)= \pm 1$ & \multirow{2}{*}{$\exp \left(\frac{2 \pi i \cdot K_{i j} \ell}{2}\right)(-1)^{i+\jmath}$} \\
\hline & $\ell$ odd & 6 & & & $\operatorname{det}(K)=K_{12}+K_{12} \bmod 2$ & \\
\hline
\end{tabular}




\begin{tabular}{|c|c|c|c|c|c|c|}
\hline \multirow{5}{*}{$\begin{array}{c}D_{2 n+1 \geq 5} \\
\pi_{1}=\mathbb{Z}_{4}\end{array}$} & $\ell \equiv 2 \bmod 4$ & 1 & \multirow{3}{*}{$\mathbb{Z}_{2}$} & \multirow{3}{*}{$\left\langle 2 \lambda_{2 n+1}\right\rangle$} & $g\left(2 \lambda_{2 n+1}, 2 \lambda_{2 n+1}\right)=1$ & \multirow{2}{*}{$\exp \left(\frac{2 \pi i \cdot\left(k \frac{\ell}{2}-2 n-1\right)}{2}\right)$} \\
\hline & $\ell \equiv 0 \bmod 4$ & 2 & & & $g\left(2 \lambda_{2 n+1}, 2 \lambda_{2 n+1}\right)= \pm 1$ & \\
\hline & $\ell$ odd & 1 & & & $g\left(2 \lambda_{2 n+1}, 2 \lambda_{2 n+1}\right)=-1$ & $\exp \left(\frac{2 \pi i \cdot(k \ell-2(2 n+1))}{2}\right)$ \\
\hline & $\ell$ even & 4 & \multirow{2}{*}{$\mathbb{Z}_{4}$} & \multirow{2}{*}{$\left\langle\lambda_{2 n+1}\right\rangle$} & $g\left(\lambda_{2 n+1}, \lambda_{2 n+1}\right)=c, c^{4}=1$ & \multirow{2}{*}{$\exp \left(\frac{2 \pi i \cdot(k \ell-(2 n+1))}{4}\right)$} \\
\hline & $\ell$ odd & 2 & & & $g\left(\lambda_{2 n+1}, \lambda_{2 n+1}\right)= \pm 1$ & \\
\hline \multirow{3}{*}{$\begin{array}{c}E_{6} \\
\pi_{1}=\mathbb{Z}_{3}\end{array}$} & $\ell \equiv 0 \bmod 3$ & 3 & \multirow{3}{*}{$\mathbb{Z}_{3}$} & \multirow{3}{*}{$\left\langle\lambda_{n}\right\rangle$} & $g\left(\lambda_{n}, \lambda_{n}\right)=c, c^{3}=1$ & \multirow{3}{*}{$\exp \left(\frac{2 \pi i \cdot(k \ell-1)}{3}\right)$} \\
\hline & $\ell \equiv 1 \bmod 3$ & 2 & & & $g\left(\lambda_{n}, \lambda_{n}\right)=1, \exp \left(\frac{2 \pi i 2}{3}\right)$ & \\
\hline & $\ell \equiv 2 \bmod 3$ & 2 & & & $g\left(\lambda_{n}, \lambda_{n}\right)=1, \exp \left(\frac{2 \pi i}{3}\right)$ & \\
\hline \multirow{2}{*}{$\begin{array}{c}E_{7} \\
\pi_{1}=\mathbb{Z}_{2}\end{array}$} & $\ell$ even & 2 & \multirow{2}{*}{$\mathbb{Z}_{2}$} & \multirow{2}{*}{$\left\langle\lambda_{n}\right\rangle$} & $g\left(\lambda_{n}, \lambda_{n}\right)= \pm 1$ & \multirow{2}{*}{$\exp \left(\frac{2 \pi i \cdot(k \ell-1)}{2}\right)$} \\
\hline & $\ell$ odd & 1 & & & $g\left(\lambda_{n}, \lambda_{n}\right)=1$ & \\
\hline
\end{tabular}

Table 2: Solutions for $R_{0}$-matrices.

The Lie algebras $E_{8}, F_{4}$ and $G_{2}$ have trivial fundamental groups and thus have no nontrivial solution. We want to emphasize once more that the choice $\Lambda_{i}=\Lambda_{R}$ always leads to a quasitriangular quantum group.

The following lemma connects our results with Lusztig's original result:

Lemma 4.7. In Lusztig's definition of a quantum group he uses the quotient

$$
\Lambda_{\text {Lusz }}^{\prime}=2 \operatorname{Cent}_{\Lambda_{R}}\left(2 \Lambda_{W}\right)
$$

This coincide with our choice $\Lambda^{\prime}=\operatorname{Cent}_{\Lambda_{R}}\left(\Lambda_{1}+\Lambda_{2}\right)$, if and only if

$$
2 \operatorname{gcd}\left(\ell, d_{i}^{\Lambda}\right)=\operatorname{gcd}\left(\ell, 2 d_{i}^{W}\right)
$$

where the $d_{i}^{\Lambda}$ denote the invariant factors of $\Lambda_{W}^{\vee} / \Lambda$ and the $d_{i}^{W}$ denote the invariant factors of $\Lambda_{W}^{\vee} / \Lambda_{W}$ (i.e., ordered root lengths).

In particular, for $\ell$ odd these choices never coincide. For $\Lambda=\Lambda_{W}, \Lambda^{\prime}=\Lambda_{\text {Lusz }}^{\prime}$ holds if and only if $2 d_{i} \mid \ell$. This is the most extreme case of divisibility and it is precisely the case appearing in logarithmic conformal field theories.

Proof. We first note that in our cases, $\Lambda^{\prime}=\operatorname{Cent}_{\Lambda_{R}}\left(\Lambda_{1}+\Lambda_{2}\right)=\operatorname{Cent}_{\Lambda_{R}}(\Lambda)$. We have

$$
\begin{aligned}
2 \operatorname{Cent}_{\Lambda_{R}}\left(2 \Lambda_{W}\right) & =2\left(\Lambda_{R} \cap \widehat{2 \Lambda_{W}}\right) \\
& =A_{R} 2\left(\Lambda_{W}^{\vee} \cap A_{W}^{-1} \frac{\ell}{2} \Lambda_{W}^{\vee}\right)=A_{R} \operatorname{Diag}\left(\frac{2 \ell}{\operatorname{gcd}\left(\ell, 2 d_{i}^{W}\right)}\right) \Lambda_{W}^{\vee}
\end{aligned}
$$

By Lemma 4.2, this coincides with $\Lambda^{\prime}$ if and only if equation (4.1) holds.

\section{$5 \quad$ Factorizability of quantum group $R$-matrices}

We first recall the definition of factorizable braided tensor categories and factorizable Hopf algebras, respectively. 
Definition 5.1 ([3]). A braided tensor category $\mathcal{C}$ is factorizable if the canonical braided tensor functor $G: \mathcal{C} \otimes \mathcal{C}^{\mathrm{op}} \rightarrow \mathcal{Z}(\mathcal{C})$ is an equivalence of categories.

In [17], Schneider gave a different characterization of factorizable Hopf algebras in terms of its Drinfeld double, leading to the following theorem:

Definition 5.2. A finite-dimensional quasitriangular Hopf algebra $(H, R)$ is called factorizable if its monodromy matrix $M:=R_{21} \cdot R \in H \otimes H$ is non-degenerate, i.e., the following linear map is bijective

$$
H^{*} \rightarrow H, \quad \phi \mapsto(\mathrm{id} \otimes \phi)(M) .
$$

Equivalently, this means we can write $M=\sum_{i} R_{1}^{i} \otimes R_{2}^{i}$ for two basis' $R_{1}^{i}, R_{2}^{j} \in H$.

Theorem 5.3. Let $(H, R)$ be a finite-dimensional quasitriangular Hopf algebra. Then the category of finite-dimensional $H$-modules $H-\bmod _{f d}$ is factorizable if and only if $(H, R)$ is a factorizable Hopf algebra.

Shimizu [18] has recently proven a number of equivalent characterizations of factorizability for arbitrary (in particular non-semisimple) braided tensor categories. Besides the two previous characterizations (equivalence to Drinfeld center and nondegeneracy of the monodromy matrix), factorizability is equivalent to the fact that the so-called transparent objects are all trivial, see Theorem 5.16 below, which will become visible during our analysis later.

\subsection{Monodromy matrix in terms of $\boldsymbol{R}_{0}$}

In order to obtain conditions for the factorizability of the quasitriangular small quantum groups $\left(u_{q}\left(\mathfrak{g}, \Lambda, \Lambda^{\prime}\right), R_{0}(f) \bar{\Theta}\right)$ as in Theorem 2.2 in terms of $\mathfrak{g}, q, \Lambda$ and $f$, we start by calculating the monodromy matrix $M:=R_{21} \cdot R \in u_{q}\left(\mathfrak{g}, \Lambda, \Lambda^{\prime}\right) \otimes u_{q}\left(\mathfrak{g}, \Lambda, \Lambda^{\prime}\right)$ in general as far as possible:

Lemma 5.4. For $R=R_{0}(f) \bar{\Theta}$ as in Theorem 2.2, the factorizability of $R$ is equivalent to the invertibility of the following complex-valued matrix $m$ with entries indexed by elements in $\mu, \nu \in \Lambda / \Lambda^{\prime}$ :

$$
m_{\mu, \nu}:=\sum_{\mu^{\prime}, \nu^{\prime} \in \Lambda / \Lambda^{\prime}} f\left(\mu-\mu^{\prime}, \nu-\nu^{\prime}\right) f\left(\nu^{\prime}, \mu^{\prime}\right)
$$

Proof. We first plug in the expressions for $R_{0}$ from Theorem 3.3 and $\bar{\Theta}$ from Theorem 2.2 and simplify:

$$
\begin{aligned}
M:= & R_{21} \cdot R=\left(R_{0}\right)_{21} \cdot \bar{\Theta}_{21} \cdot R_{0} \cdot \bar{\Theta} \\
= & \left(\sum_{\mu_{1}, \nu_{1} \in \Lambda} f\left(\mu_{1}, \nu_{1}\right) K_{\nu_{1}} \otimes K_{\mu_{1}}\right)\left(\sum_{\beta_{1} \in \Lambda_{R}^{+}}(-1)^{\operatorname{tr} \beta_{1}} q_{\beta_{1}} \sum_{b_{1} \in B_{\beta_{2}}} b_{1}^{*+} \otimes b_{1}^{-}\right) \\
& \times\left(\sum_{\mu_{2}, \nu_{2} \in \Lambda} f\left(\mu_{2}, \nu_{2}\right) K_{\mu_{2}} \otimes K_{\nu_{2}}\right)\left(\sum_{\beta_{2} \in \Lambda_{R}^{+}}(-1)^{\operatorname{tr} \beta_{2}} q_{\beta_{2}} \sum_{b_{2} \in B_{\beta_{2}}} b_{2}^{-} \otimes b_{2}^{*+}\right) \\
= & \sum_{\beta_{1}, \beta_{2} \in \Lambda_{R}^{+}}(-1)^{\operatorname{tr} \beta_{1}+\beta_{2}} q_{\beta_{1}} q_{\beta_{2}}\left(\sum_{\mu_{1}, \mu_{2}, \nu_{1}, \nu_{2} \in \Lambda} f\left(\mu_{1}, \nu_{1}\right) f\left(\mu_{2}, \nu_{2}\right) q^{\beta_{1}\left(\nu_{2}-\mu_{2}\right)} K_{\nu_{1}+\mu_{2}} \otimes K_{\mu_{1}+\nu_{2}}\right) \\
& \times\left(\sum_{b_{1} \in B_{\beta_{1}, b_{2} \in B_{\beta_{2}}}} b_{1}^{*+} b_{2}^{-} \otimes b_{1}^{-} b_{2}^{*+}\right),
\end{aligned}
$$


where $\Lambda_{R}^{+}=\mathbb{N}_{0}[\Delta]$. The last equation holds since $b_{1}^{-} \in u_{\beta_{1}}^{-}$and hence fulfills $K_{\nu_{2}} b_{1}^{-}=$ $q^{-\beta_{1} \nu_{2}} b_{1}^{-} K_{\nu_{2}}$ and similarly for $b_{1}^{*+}$. We have two triangular decompositions

$$
u_{q}=u_{q}^{0} u_{q}^{-} u_{q}^{+}, \quad u_{q}=u_{q}^{0} u_{q}^{+} u_{q}^{-},
$$

and the $\Lambda_{R}^{+}$-gradation on $u_{q}^{ \pm}$induces a gradation

$$
u_{q} \otimes u_{q} \cong \bigoplus_{\beta_{1}, \beta_{2}}\left(u^{0} \otimes u^{0}\right)\left(u_{q_{\beta_{1}}}^{+} u_{q_{\beta_{2}}}^{-} \otimes u_{q_{\beta_{1}}}^{-} u_{q_{\beta_{2}}}^{+}\right) \text {. }
$$

The factorizability of $R$ is equivalent to the invertibility of $M$ interpreted as a matrix indexed by the PBW basis. The grading implies a block matrix form of $M$, so the invertibility $M$ is equivalent to the invertibility of $M^{\beta_{1}, \beta_{2}} \in\left(u_{q} \otimes u_{q}\right)_{\left(\beta_{1}, \beta_{2}\right)}$ for every $\beta_{1}, \beta_{2} \in \Lambda_{R}^{+}$as follows

$$
\begin{aligned}
M^{\beta_{1}, \beta_{2}}:= & \left(\sum_{\mu_{1}, \mu_{2}, \nu_{1}, \nu_{2} \in \Lambda} f\left(\mu_{1}, \nu_{1}\right) f\left(\mu_{2}, \nu_{2}\right) q^{\beta_{1}\left(\nu_{2}-\mu_{2}\right)} K_{\nu_{1}+\mu_{2}} \otimes K_{\mu_{1}+\nu_{2}}\right) \\
& \times\left(\sum_{b_{1} \in B_{\beta_{1}, b_{2} \in B_{\beta_{2}}}} b_{1}^{*+} b_{2}^{-} \otimes b_{1}^{-} b_{2}^{*+}\right) .
\end{aligned}
$$

Since the second sum in $M^{\beta_{1}, \beta_{2}}$ runs over a basis in $u_{q}^{+}{ }_{\beta_{1}} u_{q}^{-} \beta_{2} \otimes u_{q}^{-}{ }_{\beta_{1}} u_{q}^{+}{ }_{\beta_{2}}$, the invertibility of $M$ is equivalent to the invertibility for all $\beta_{1} \in \Lambda_{R}^{+}$the following element:

$$
\begin{aligned}
M_{0}^{\beta_{1}} & :=\sum_{\mu_{1}, \mu_{2}, \nu_{1}, \nu_{2} \in \Lambda / \Lambda^{\prime}} q^{\beta_{1}\left(\nu_{2}-\mu_{2}\right)} f\left(\mu_{1}, \nu_{1}\right) f\left(\mu_{2}, \nu_{2}\right) K_{\nu_{1}+\mu_{2}} \otimes K_{\mu_{1}+\nu_{2}} \\
& =\sum_{\mu, \nu \in \Lambda / \Lambda^{\prime}} K_{\nu} \otimes K_{\mu} \cdot\left(\sum_{\mu^{\prime}, \nu^{\prime} \in \Lambda / \Lambda^{\prime}} q^{\beta_{1}\left(\mu^{\prime}-\nu^{\prime}\right)} f\left(\mu-\mu^{\prime}, \nu-\nu^{\prime}\right) f\left(\nu^{\prime}, \mu^{\prime}\right)\right) .
\end{aligned}
$$

Since $K_{\nu} \otimes K_{\mu}$ is a vector space basis of $u_{q}^{0} \otimes u_{q}^{0}=\mathbb{C}\left[\Lambda / \Lambda^{\prime}\right] \otimes \mathbb{C}\left[\Lambda / \Lambda^{\prime}\right]$, this in turn is equivalent to the invertibility of the following family of matrices $m^{\beta_{1}}$ for all $\beta_{1} \in \Lambda_{R}^{+}$with rows/columns indexed by elements in $\mu, \nu \in \Lambda / \Lambda^{\prime}$ :

$$
m_{\mu, \nu}^{\beta_{1}}:=\sum_{\mu^{\prime}, \nu^{\prime} \in \Lambda / \Lambda^{\prime}} f\left(\mu-\mu^{\prime}, \nu-\nu^{\prime}\right) f\left(\nu^{\prime}, \mu^{\prime}\right) q^{\beta_{1}\left(\mu^{\prime}-\nu^{\prime}\right)} .
$$

We now use the fact that $R$ was indeed an $R$-matrix. By property (2.1) in Theorem 2.2 we have

$$
m_{\mu, \nu}^{\beta_{1}}=\sum_{\mu^{\prime}, \nu^{\prime} \in \Lambda / \Lambda^{\prime}} f\left(\mu-\mu^{\prime}, \nu-\nu^{\prime}\right) f\left(\nu^{\prime}+\beta_{1}, \mu^{\prime}\right) q^{-\beta_{1} \nu^{\prime}}
$$

Since the invertibility of a matrix $m_{\mu, \nu}$ is equivalent to the invertibility of any matrix $m_{\mu, \nu+\beta_{1}}$, we may substitute $\nu^{\prime} \mapsto \nu^{\prime}+\beta_{1}, \nu \mapsto \nu+\beta_{1}$, pull the constant factor $q^{-\beta_{1}^{2}}$ in front (which also does not affect invertibility) and hence eliminate the first $\beta_{1}$ from the condition. Hence the invertibility of $R$ is equivalent to the invertibility of the following family of matrices $\tilde{m}^{\beta_{1}}$ for all $\beta_{1} \in \Lambda_{R}^{+}$:

$$
\tilde{m}_{\mu, \nu}^{\beta_{1}}:=\sum_{\mu^{\prime}, \nu^{\prime} \in \Lambda / \Lambda^{\prime}} f\left(\mu-\mu^{\prime}, \nu-\nu^{\prime}\right) f\left(\nu^{\prime}, \mu^{\prime}\right) q^{-\beta_{1} \nu^{\prime}} .
$$


We may now use the same procedure to eliminate the second $\beta_{1}$, hence the invertibility of $R$ is equivalent to the invertibility of the following matrix with rows/columns induced by elements in $\mu, \nu \in \Lambda / \Lambda^{\prime}$ :

$$
m_{\mu, \nu}:=\sum_{\mu^{\prime}, \nu^{\prime} \in \Lambda / \Lambda^{\prime}} f\left(\mu-\mu^{\prime}, \nu-\nu^{\prime}\right) f\left(\nu^{\prime}, \mu^{\prime}\right) .
$$

This was the assertion we wanted to prove.

Definition 5.5. Let $g: G_{1} \times G_{2} \rightarrow \mathbb{C}^{\times}$be a group pairing. It induces a symmetric form on the product $G_{1} \times G_{2}$ we denote by $\operatorname{Sym}(g)$ :

$$
\begin{aligned}
\operatorname{Sym}(g): & \left(G_{1} \times G_{2}\right)^{\times 2} \longrightarrow \mathbb{C}^{\times}, \\
& \left(\left(\mu_{1}, \mu_{2}\right),\left(\nu_{1}, \nu_{2}\right)\right) \longmapsto g\left(\mu_{1}, \nu_{2}\right) g\left(\nu_{1}, \mu_{2}\right) .
\end{aligned}
$$

Lemma 5.6. If $g: G_{1} \times G_{2} \rightarrow \mathbb{C}^{\times}$is a perfect pairing of abelian groups, then the symmetric form $\operatorname{Sym}(g)$ is perfect.

Proof. By assumption, $g \times g$ defines an isomorphism between $G_{1} \times G_{2}$ to $\widehat{G_{2}} \times \widehat{G_{1}}$. The symmetric form $\operatorname{Sym}(g)$ is given by the composition of this isomorphism with the canonical isomorphism $\widehat{G_{2}} \times \widehat{G_{1}} \cong \widehat{G_{1} \times G_{2}}$. This proves the claim.

Consider for a finite abelian group $G$ and subgroups $G_{1}, G_{2} \leq G$ the canonical exact sequence

$$
0 \rightarrow G_{1} \cap G_{2} \rightarrow G_{1} \times G_{2} \rightarrow G_{1}+G_{2} \rightarrow 0 .
$$

For $\mu \in G_{1}+G_{2}$, we denote its fiber by

$$
\left(G_{1} \times G_{2}\right)_{\mu}:=\left\{\left(\mu_{1}, \mu_{2}\right) \in G_{1} \times G_{2} \mid \mu_{1}+\mu_{2}=\mu\right\} .
$$

Moreover, we define

$$
\begin{aligned}
& \operatorname{Rad}:=\left\{\left(\mu_{1}, \mu_{2}\right) \in G_{1} \times G_{2} \mid \operatorname{Sym}(\hat{f})\left(\left(\mu_{1}, \mu_{2}\right), x\right)=1 \forall x \in\left(G_{1} \times G_{2}\right)_{0}\right\}, \\
& \operatorname{Rad}_{\mu}:=\operatorname{Rad} \cap\left(G_{1} \times G_{2}\right)_{\mu}, \\
& \operatorname{Rad}_{0}^{\perp}:=\left\{\mu_{1}+\mu_{2} \in G \mid\left(\mu_{1}, \mu_{2}\right) \in \operatorname{Rad}\right\} .
\end{aligned}
$$

Lemma 5.7. We have two split exact sequences:

$$
\begin{aligned}
& 0 \rightarrow \operatorname{Rad}_{0} \rightarrow \operatorname{Rad} \rightarrow \operatorname{Rad}_{0}^{\perp} \rightarrow 0, \\
& 0 \rightarrow \operatorname{Rad}_{0}^{\perp} \rightarrow G \rightarrow \operatorname{Rad}_{0} \rightarrow 0 .
\end{aligned}
$$

Proof. The first sequence is exact by definition of the three groups. Moreover, we know

$$
\operatorname{Rad}=\operatorname{ker}(\hat{\iota} \circ \operatorname{Sym}(\hat{f})) \cong \operatorname{ker}(\hat{\iota})=\operatorname{im}(\hat{\pi}) \cong \hat{G} \cong G,
$$

where $\hat{\iota}, \hat{\pi}$ denote the duals of the inclusion and projection in (5.1). In Example 5.11 we will see that in the case $G_{1}=G_{2}=G, \hat{f}$ symmetric, $\operatorname{Rad}_{0}$ is the 2-torsion subgroup of $G$, and the second map in the second exact sequence is just the projection, hence both diagrams split in this case. If $\hat{f}$ is asymmetric, we will see in Section 5.3 that $\operatorname{Rad}_{0}$ is isomorphic to $\mathbb{Z}_{2}^{k}$ for some $k \geq 2$, thus

$$
\operatorname{Rad}_{0}^{\perp} \longrightarrow \operatorname{Rad}, \quad x \longmapsto \sum_{\tilde{x} \in \operatorname{Rad}_{x}} \tilde{x}
$$

is a section of the first exact sequence. Here we used that the sum over all elements in $\mathbb{Z}_{2}^{k}$ vanishes. Again, it follows that both diagrams split. Finally, if $G_{1} \neq G_{2}$ (i.e., in the case $D_{2 n}$ ), then $\hat{f}=q^{-(\cdot, \cdot)}$ on $G_{1} \cap G_{2}$. By the same argument as in Example 5.11, $\operatorname{Rad}_{0}$ is the 2-torsion subgroup of $G_{1} \cap G_{2}$. But we have $G \cong G_{1} \cap G_{2} \times \pi_{1}$ in this case, hence both sequences split. 
Corollary 5.8. Using the projection $\alpha: G \rightarrow \operatorname{Rad}_{0}^{\perp}$ and the inclusion $\beta: \operatorname{Rad}_{0}^{\perp} \rightarrow \operatorname{Rad}$ from the above lemma, we can define a symmetric form on $G$ :

$$
\begin{aligned}
& \operatorname{Sym}_{G}(\hat{f}): G \times G \longrightarrow \mathbb{C}^{\times}, \\
&(\mu, \nu) \longmapsto \operatorname{Sym}(\hat{f})(\beta \circ \alpha(\mu), \beta \circ \alpha(\nu)) .
\end{aligned}
$$

Moreover, we have $\operatorname{Rad}\left(\operatorname{Sym}_{G}(\hat{f})\right) \cong \operatorname{Rad}_{0}$.

Theorem 5.9. We have shown in Theorem 2.2 and Lemma 3.5 that the assumption that $R=$ $R_{0}(f) \bar{\Theta}$ is an $R$-matrix is equivalent to the existence of subgroups $G_{1}, G_{2} \subset \Lambda / \Lambda^{\prime}$ of same order some $d\left|\Lambda_{R} / \Lambda^{\prime}\right|$ and $f$ restricting up to a scalar to a non-degenerate pairing $\hat{f}: G_{1} \times G_{2} \rightarrow \mathbb{C}^{\times}$ and $f$ vanishes otherwise.

In this notation the matrix $m$ as defined in the previous lemma can be rewritten as

$$
m_{\mu, \nu}=\frac{1}{d^{2}\left|\Lambda_{R} / \Lambda^{\prime}\right|^{2}} \sum_{\substack{\tilde{\mu} \in\left(G_{1} \times G_{2}\right)_{\mu} \\ \tilde{\nu} \in\left(G_{1} \times G_{2}\right)_{\nu}}} \operatorname{Sym}(\hat{f})(\tilde{\mu}, \tilde{\nu}) .
$$

It is invertible if and only if $\operatorname{Rad}_{0}=0$. In this case,

$$
m_{\mu, \nu}=\frac{\left|G_{1} \cap G_{2}\right|}{d^{2}\left|\Lambda_{R} / \Lambda^{\prime}\right|^{2}} \operatorname{Sym}_{G}(\hat{f}) .
$$

We first note that $\operatorname{Rad}_{0}=0$ implies $\operatorname{Rad}_{0}^{\perp}=G$ and thus $G=G_{1}+G_{2}$. Together with Corollary 3.6 this implies

\section{Corollary 5.10.}

$$
\Lambda^{\prime}=\operatorname{Cent}_{\Lambda_{R}}(\Lambda)
$$

Before we proof the theorem, we first give a simple example:

Example 5.11. Let $G_{1}=G_{2}=G$ (correspondingly $\Lambda_{1}=\Lambda_{2}=\Lambda$ ) and assume $\hat{f}$ is symmetric non-degenerate, then the radical measures 2-torsion:

$$
\operatorname{Rad}\left(\operatorname{Sym}_{G}(\hat{f})\right) \cong \operatorname{Rad}_{0}=\{\mu \in G \mid 2 \mu=0\}
$$

Again, this is the only case appearing for cyclic fundamental groups. Hence in all cases except $\mathfrak{g}=D_{2 n}$ factorizability is equivalent to $\left|\Lambda / \Lambda^{\prime}\right|$ being odd.

Proof of Theorem 5.9. The first part of the theorem follows by applying Lemma 3.5 to the matrix $m$ as given in the previous lemma. Now, assume that $m$ is invertible. We must have $G=G_{1}+G_{2}$, otherwise the matrix has zero-columns and rows, differently formulated: the fibers $\left(G_{1} \times G_{2}\right)_{\mu}$ in the short exact sequence must be non-empty for all $\mu \in G$. If on the other hand, $\operatorname{Rad}_{0}=0$, then $\operatorname{Rad}_{0}^{\perp}=G$ and thus $G_{1}+G_{2}=G$ must also hold, thus we assume this from now on. By the short exact sequence the fiber $\left(G_{1} \times G_{2}\right)_{0} \cong G_{1} \cap G_{2}$, other fibers are of the explicit form $\tilde{\mu}+\left(G_{1} \times G_{2}\right)_{0}$ for some choice of representative $\tilde{\mu}$. Therefore,

$$
\begin{aligned}
m_{\mu, \nu} & =\frac{1}{d^{2}\left|\Lambda_{R} / \Lambda^{\prime}\right|^{2}} \sum_{\substack{\tilde{\mu} \in\left(G_{1} \times G_{2}\right)_{\mu} \\
\tilde{\nu} \in\left(G_{1} \times G_{2}\right)_{\nu}}} \operatorname{Sym}(\hat{f})(\tilde{\mu}, \tilde{\nu}) \\
& =\frac{1}{d^{2}\left|\Lambda_{R} / \Lambda^{\prime}\right|^{2}} \sum_{\tilde{\nu} \in\left(G_{1} \times G_{2}\right)_{\nu}} \operatorname{Sym}(\hat{f})(\tilde{\mu}, \tilde{\nu}) \sum_{\tilde{\eta} \in\left(G_{1} \times G_{2}\right)_{0}} \operatorname{Sym}(\hat{f})(\tilde{\eta}, \tilde{\nu})
\end{aligned}
$$




$$
=\left.\frac{\left|G_{1} \cap G_{2}\right|}{d^{2}\left|\Lambda_{R} / \Lambda^{\prime}\right|^{2}} \sum_{\tilde{\nu} \in\left(G_{1} \times G_{2}\right)_{\nu}} \operatorname{Sym}(\hat{f})(\tilde{\mu}, \tilde{\nu}) \cdot \delta_{\operatorname{Sym}(f)(\tilde{\nu},-)}\right|_{G_{1} \cap G_{2}}=1=(*) .
$$

Fix as above a representative $\tilde{\nu}$ of the fiber of $\nu$, i.e., $\tilde{\nu} \in\left(G_{1} \times G_{2}\right)_{\nu}$ such that $\left.\operatorname{Sym}(f)\left(\tilde{\nu},{ }_{-}\right)\right|_{G_{1} \cap G_{2}}$ $=1$ holds. Two elements fulfilling this property differ by an element in the subgroup $\operatorname{Rad}_{0} \leq$ $G_{1} \cap G_{2}$, thus

$$
\begin{aligned}
& (*)=\left.\frac{\left|G_{1} \cap G_{2}\right|}{d^{2}\left|\Lambda_{R} / \Lambda^{\prime}\right|^{2}} \operatorname{Sym}(\hat{f})(\tilde{\mu}, \tilde{\nu}) \sum_{\tilde{\xi} \in \operatorname{Rad}_{0}} \operatorname{Sym}(\hat{f})(\tilde{\xi}, \tilde{\nu}) \cdot \delta_{\operatorname{Sym}(f)(\tilde{\nu},-)}\right|_{G_{1} \cap G_{2}}=1
\end{aligned}
$$

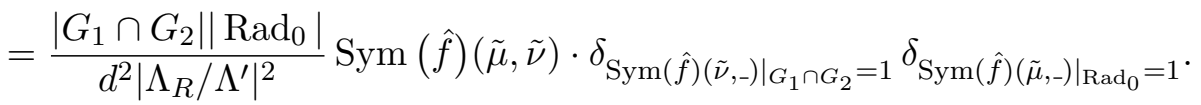

Since $m$ is symmetric, we have

$$
\begin{aligned}
& m_{\mu, \nu}=\left.\frac{\left|G_{1} \cap G_{2}\right|\left|\operatorname{Rad}_{0}\right|}{d^{2}\left|\Lambda_{R} / \Lambda^{\prime}\right|^{2}} \operatorname{Sym}(\hat{f})(\tilde{\mu}, \tilde{\nu}) \cdot \delta_{\operatorname{Sym}(\hat{f})(\tilde{\nu},-)}\right|_{G_{1} \cap G_{2}}=1 \\
&=\left.\frac{\left|G_{1} \cap G_{2}\right|\left|\operatorname{Rad}_{0}\right|}{d^{2}\left|\Lambda_{R} / \Lambda^{\prime}\right|^{2}} \operatorname{Sym}_{G}(\hat{f})(\tilde{\mu})(\mu, \nu)\right|_{G_{1} \cap G_{2}}=1 \\
& \operatorname{Rad}_{\mu} \neq \varnothing \delta_{\operatorname{Rad}_{\nu} \neq \varnothing}
\end{aligned}
$$

and this is invertible if an only if $\operatorname{Rad}_{0} \cong \operatorname{Rad}\left(\operatorname{Sym}_{G}(\hat{f})\right)=0$.

\subsection{Factorizability for symmetric $R_{0}(f)$}

For $R_{0}=\sum_{\mu, \nu} f(\mu, \nu) K_{\mu} \otimes K_{\nu}$ being the Cartan part of an $R$-matrix, assume that $\hat{f}=|G| f$ on $G$ is symmetric. We have shown in Example 5.11 that factorizability is equivalent to $|G|$ being odd.

We now want to give a necessary and sufficient condition for this:

Lemma 5.12. Let $\Lambda_{R} \subseteq \Lambda \subseteq \Lambda_{W}$ be an arbitrary intermediate lattice for a certain irreducible root system. Then the order of the group $G=\Lambda / \operatorname{Cent}_{\Lambda_{R}}(\Lambda)$ is odd if and only if both of the following conditions are satisfied:

1) $\left|\Lambda / \Lambda_{R}\right|$ is odd,

2) $\ell$ is either odd or $\left(\ell \equiv 2 \bmod 4, \mathfrak{g}=B_{n}, \Lambda=\Lambda_{R}\right)$ including $A_{1}$.

Proof. We saw that in all our cases, there exists an isomorphism

$$
\Lambda / \Lambda_{R} \cong \operatorname{Cent}_{\Lambda}\left(\Lambda_{R}\right) / \operatorname{Cent}_{\Lambda_{R}}(\Lambda) .
$$

Moreover, from Lemma 4.2 we know that $\left|\Lambda / \operatorname{Cent}_{\Lambda}\left(\Lambda_{R}\right)\right|=\operatorname{det}\left(D_{\ell}\right)$, where $D_{\ell}$ was the diagonal matrix $\left.\operatorname{Diag}\left(\frac{\ell}{\operatorname{gcd}\left(\ell, d_{i}^{\Lambda}\right)}\right)\right)$ with $d_{i}^{\Lambda}$ being the invariant factors of the lattice $\Lambda$ (i.e., the diagonal entries of the Smith normal form of a basis matrix of $\Lambda$ ). Thus,

$$
\begin{aligned}
|G| & =\left|\Lambda / \operatorname{Cent}_{\Lambda_{R}}(\Lambda)\right|=\left|\Lambda / \operatorname{Cent}_{\Lambda}\left(\Lambda_{R}\right)\right|\left|\operatorname{Cent}_{\Lambda}\left(\Lambda_{R}\right) / \operatorname{Cent}_{\Lambda_{R}}(\Lambda)\right| \\
& =\left|\Lambda / \operatorname{Cent}_{\Lambda}\left(\Lambda_{R}\right)\right|\left|\Lambda / \Lambda_{R}\right|=\operatorname{det}\left(D_{\ell}\right)\left|\Lambda / \Lambda_{R}\right|=\prod_{i=1}^{n} \frac{\ell}{\operatorname{gcd}\left(\ell, d_{i}^{\Lambda}\right)}\left|\Lambda / \Lambda_{R}\right| .
\end{aligned}
$$

Clearly, this term is odd if $\ell$ and $\left|\Lambda / \Lambda_{R}\right|$ are odd. In the case $\left(\ell \equiv 2 \bmod 4, \mathfrak{g}=B_{n}, \Lambda=\Lambda_{R}\right)$, the Smith normal form $S_{R}$ of the basis matrix $A_{R}$ is given by $2 \cdot$ id. Thus, $|G|$ is odd in this case. On the other hand, let $|G|$ be odd:

We first consider the case $\ell$ even. A necessary condition for $\left|\Lambda / \Lambda^{\prime}\right|$ odd is that the multiplicity $m_{\ell}$ of the prime 2 in $\prod_{i=1}^{n} \frac{\ell}{\operatorname{gcd}\left(\ell, d_{i}^{\Lambda}\right)}$ is at most the multiplicity $m_{\pi_{1}}$ of the prime 2 in $\left|\pi_{1}\right|$. We check this condition for rank $n>1$ : 
- For $\mathfrak{g}$ simply-laced (or triply-laced $\mathfrak{g}=G_{2}$ ) we have all $d_{i}=1$, hence $n \mid m_{\ell}$ (equality for $\ell=2 \bmod 4$ ). The cases $D_{n}$ with $m_{\pi_{1}}=2$ have rank $n \geq 4$, all others except $A_{n}$ have $m_{\pi_{1}}=0,1$, so the necessary condition $m_{\ell} \leq m_{\pi_{1}}$ is never fulfilled. The cases $A_{n}$ have $2^{m_{\pi_{1}} \mid(n+1)} \leq\left(m_{\ell}+1\right) \stackrel{!}{\leq}\left(m_{\pi_{1}}+1\right)$ which can only be true in rank $n=1$ treated above.

- For $\mathfrak{g}$ doubly-laced of rank $n>1$, we always have always $m_{\pi_{1}}=0,1$ but $m_{\ell}$ can be considerably smaller than above, namely for $\ell=2 \bmod 4$ equal to the number of short simple roots $d_{\alpha_{i}}=1$ (otherwise $m_{\ell}$ again increases by $n$ for every factor 2 in $\ell$ ), hence the necessary condition $m_{\ell} \leq m_{\pi_{1}}$ can be fulfilled only for $B_{n}$ (which would also include $A_{1}$ above for $n=1$ ). More precisely, since $m_{\ell}=m_{\pi_{1}}$ and the decomposition for $\Lambda / \Lambda^{\prime}$ has an additional factor $\left|\Lambda / \Lambda_{R}\right|$, it can only be odd for $\Lambda=\Lambda_{R}$.

On the other hand, if $\ell$ is odd, then the whole product term is odd. But since $|G|$ was assumed to be odd, also $\left|\Lambda / \Lambda^{\prime}\right|$ must be odd.

Corollary 5.13. Let $\Lambda=\Lambda_{R}$. In the previous section we have seen that $\hat{f}=q^{-(\cdot, \cdot)}$ gives always an $R$-matrix in this case. By the proof of the previous lemma, we have

$$
\operatorname{Rad}_{0} \cong \prod_{i=1}^{n} \mathbb{Z}_{\operatorname{gcd}\left(2, \frac{\ell}{\operatorname{gcd}\left(\ell, d_{i}^{R}\right)}\right)},
$$

where the $d_{i}^{R}$ denote the invariant factors of $\Lambda_{W}^{\vee} / \Lambda_{R}$.

\subsection{Factorizability for $D_{2 n}, R_{0}$ antisymmetric}

The split case $\mathfrak{g}=D_{2 n}, G=G_{1} \times G_{2}$ is clearly factorizable, so the only remaining case for which we have to check factorizabilty is $\mathfrak{g}=D_{2 n}, \Lambda=\Lambda_{W}$ for $\hat{f}$ being not symmetric. We know that in this case, the corresponding form $g$ on $\Lambda / \Lambda_{R}$ is uniquely defined by a $2 \times 2$-matrix $K \in \mathfrak{g l}\left(2, \mathbb{F}_{2}\right)$, s.t. $g\left(\lambda_{2(n-1)+i}, \lambda_{2(n-1)+j}\right)=\exp \left(\frac{2 \pi i K_{i j}}{2}\right)$ for $i, j \in\{1,2\}$. From this we see that if $g$ is not symmetric, it must be antisymmetric, i.e., $g(\mu, \nu)=g(\nu, \mu)^{-1}$. Thus, the following lemma applies in this case, and hence there are no factorizable $R$-matrices for $D_{2 n}, \Lambda=\Lambda_{W}$.

Lemma 5.14. For $\mathfrak{g}$ simply-laced and $\Lambda=\Lambda_{W}$, let $\hat{f}=q^{-(\cdot, \cdot)} g: G \times G \rightarrow \mathbb{C}^{\times}$be a non-degenerate form as in Theorem 3.3 and Lemma 3.5, s.t. the form $g: \pi_{1} \times \pi_{1} \rightarrow \mathbb{C}^{\times}$is asymmetric. Then,

$$
\operatorname{Rad}_{0} \cong \bigoplus_{i=1}^{n} \mathbb{Z}_{\operatorname{gcd}\left(2, \ell d_{i}^{R}\right)},
$$

where the $d_{i}^{R}$ denote the invariant factors of $\pi_{1}$. In particular, $\operatorname{Rad}_{0}=0$ holds if and only if $\operatorname{gcd}\left(2, \ell\left|\pi_{1}\right|\right)=1$.

Proof. We recall the definition of $\operatorname{Rad}_{0}\left(\operatorname{Sym}_{G}(\hat{f})\right)$ in this case:

$$
\begin{aligned}
\operatorname{Rad}_{0}\left(\operatorname{Sym}_{G}(\hat{f})\right) & =\left\{\mu \in G \mid f(\nu, \mu)^{-1}=f(\mu, \nu) \forall \nu \in G\right\} \\
& =\left\{\mu \in G \mid q^{(\nu, \mu)} g(\nu, \mu)^{-1}=q^{-(\mu, \nu)} g(\mu, \nu) \forall \nu \in G\right\} \\
& =\left\{\mu \in G \mid q^{(\nu, \mu)}=q^{-(\mu, \nu)} \forall \nu \in G\right\} \\
& =\left\{\mu \in G \mid q^{(2 \mu, \nu)}=1 \forall \nu \in G\right\} \\
& =\left\{\mu \in G \mid 2 \mu \in \operatorname{Cent}_{2 \Lambda_{W}}\left(\Lambda_{W}\right) / 2 \operatorname{Cent}_{\Lambda_{R}}\left(\Lambda_{W}\right)\right\}=(*) .
\end{aligned}
$$

For $\mathfrak{g}$ is simply-laced, we have $\Lambda_{W}=\Lambda_{W}^{\vee}$, thus

$$
\begin{aligned}
(*) & \cong \operatorname{Cent}_{2 \Lambda_{W}}\left(\Lambda_{W}\right) / 2 \operatorname{Cent}_{\Lambda_{R}}\left(\Lambda_{W}\right)=\left(2 \Lambda_{W} \cap \ell A_{R} \Lambda_{W}\right) / 2 \ell A_{R} \Lambda_{W} \\
& =P_{R} \operatorname{Diag}\left(\operatorname{lcm}\left(2, \ell d_{i}^{R}\right)\right) \Lambda_{W} / P_{R} 2 \ell S_{R} \Lambda_{W}=\Lambda_{W} / \operatorname{Diag}\left(\operatorname{gcd}\left(2, \ell d_{i}^{R}\right)\right) \Lambda_{W} .
\end{aligned}
$$

This proves the claim. 


\subsection{Transparent objects in non-factorizable cases}

In this section, we determine the transparent objects in the representation category of $u_{q}(\mathfrak{g}, \Lambda)$ with our $R$-matrix given by $R_{0} \bar{\Theta}$ and $R_{0}=\frac{1}{\left|\Lambda / \Lambda^{\prime}\right|} \sum_{\mu, \nu \in \Lambda / \Lambda^{\prime}} \hat{f}$ with $\hat{f}$ a group pairing $\Lambda_{1} / \Lambda^{\prime} \times$ $\Lambda_{2} / \Lambda^{\prime} \rightarrow \mathbb{C}^{\times}$

Definition 5.15. Let $\mathcal{C}$ be a braided monoidal category with braiding $c$. An object $V \in \mathcal{C}$ is called transparent if the double braiding $c_{W, V} \circ c_{V, W}$ is the identity on $V \otimes W$ for all $W \in \mathcal{C}$.

The following theorem by Shimizu gives a very important characterization of factorizable categories:

Theorem 5.16 ([18, Theorem 1.1]). A braided finite tensor category is factorizable if and only if the transparent objects are direct sums of finitely many copies of the unit object.

Corollary 5.17. In particular, for a Hopf algebra $H$ the representation category $H-\bmod _{f d}$ is factorizable if and only if the transparent objects are multiples of the trivial representation and vice versa.

Since in our cases $\Lambda_{1} \neq \Lambda_{2}$ can only appear in $D_{2 n}$, and we know those are factorizable, we shall in the following restrict ourselves to the case $\Lambda_{1}=\Lambda_{2}=\Lambda$. The proof below works also in the more general case, but requires more notation. As usual we first reduce the Hopf algebra question to the group ring and then solve the group theoretical problem.

Lemma 5.18. If a $u_{q}(\mathfrak{g})$-module $V$, with a highest-weight vector $v$ and $K_{\mu} v=\chi\left(K_{\mu}\right) v$, is a transparent object, then necessarily the 1-dimensional $\Lambda / \Lambda^{\prime}$-module $\mathbb{C}_{\chi}$ is a transparent object over the Hopf algebra $\mathbb{C}\left[\Lambda / \Lambda^{\prime}\right]$ with $R$-matrix $R_{0}$. If $V$ is 1-dimensional, then $V$ is transparent if and only if $\mathbb{C}_{\chi}$ is.

Proof. Let $V$ be transparent. For every $\psi: \Lambda / \Lambda^{\prime} \rightarrow \mathbb{C}^{\times}$we have another finite-dimensional module $W:=u_{q}(\mathfrak{g}) \otimes_{u_{q}(\mathfrak{g})}+\mathbb{C}_{\psi}$ with highest weight vector $w=1 \otimes 1_{\psi}$ which we can test this assumption against

$$
c^{2}: V \otimes W \rightarrow W \otimes V \rightarrow V \otimes W .
$$

We calculate the effect of $c^{2}$ on the highest-weight vectors $v \otimes w$ :

$$
c^{2}(v \otimes w)=\tau_{W \otimes V} R_{0} \bar{\Theta} \tau_{V \otimes W} R_{0} \bar{\Theta}(v \otimes w) .
$$

Because $v, w$ were assumed highest-weight vectors, the $\bar{\Theta}$ act trivially. Hence follows that $\mathbb{C}_{\chi}, \mathbb{C}_{\psi}$ have a trivial double braiding over the Hopf algebra $\mathbb{C}\left[\Lambda / \Lambda^{\prime}\right]$ with $R$-matrix $R_{0}$. Because we could achieve this result for any $\psi$ this means that $\mathbb{C}_{\chi}$ is transparent as asserted.

Now, let $V=\mathbb{C}_{\chi}$ be 1 -dimensional over $u_{q}(\mathfrak{g})$ and transparent over $\mathbb{C}\left[\Lambda / \Lambda^{\prime}\right]$, and let $w$ be any element in any module $W$, then again the two $\Theta$ act trivially, one time because $v=1_{\chi}$ is a highest weight vector, and one time because it is also a lowest weight vector. But if the double-braiding of $v=1_{\chi}$ with any element $w$ is trivial, then $V=\mathbb{C}_{\chi}$ is already transparent over $u_{q}(\mathfrak{g})$.

Lemma 5.19. $\mathbb{C}_{\chi}$ is a transparent object over the Hopf algebra $\mathbb{C}\left[\Lambda / \Lambda^{\prime}\right]$ with $R$-matrix $R_{0}$ iff it is an $f$-transformed of the radical of $\operatorname{Sym}_{G}(\hat{f})$, i.e.,

$$
\chi(\mu)=f(\mu, \xi), \quad \xi \in \operatorname{Rad}_{0} .
$$


Proof. Since $f$ is nondegenerate, we can assume $\chi(\mu)=f(\mu, \xi)$ and wish to prove $\mathbb{C}_{\chi}$ is transparent iff $\xi \in \operatorname{Rad}_{0}$. We test transparency against any module $\mathbb{C}_{\psi}$ and also write $\psi(\mu)=$ $f(\lambda, \mu)$ (note the order of the argument). We evaluate the double-braiding on $1_{\chi} \otimes 1_{\psi}$ and get the following scalar factor, which needs to be $=1$ for all $\psi$ in order to make $\mathbb{C}_{\chi}$ transparent:

$$
\begin{aligned}
\frac{1}{|G|^{2}} \sum_{\mu, \nu} \chi(\mu) \psi(\nu) \sum_{\substack{\mu_{1}+\mu_{2}=\mu \\
\nu_{1}+\nu_{2}=\mu}} \operatorname{Sym}(\hat{f})\left(\left(\mu_{1}, \mu_{2}\right),\left(\nu_{1}, \nu_{2}\right)\right) \\
\quad=\frac{1}{|G|^{2}} \sum_{\mu, \nu} f(\mu, \xi) f(\lambda, \nu) \sum_{\substack{\mu_{1}+\mu_{2}=\mu \\
\nu_{1}+\nu_{2}=\mu}} f\left(\mu_{1}, \nu_{1}\right) f\left(\nu_{2}, \mu_{2}\right) \\
=\frac{1}{|G|^{2}} \sum_{\mu, \nu} f(\mu, \xi) f(\lambda, \nu) \sum_{\nu_{1}, \mu_{1}} f\left(\mu_{1}, \nu_{1}\right) f(\nu, \mu) f^{-1}\left(\nu_{1}, \mu\right) f^{-1}\left(\nu, \mu_{1}\right) f\left(\nu_{1}, \mu_{1}\right) \\
=\frac{1}{|G|} \sum_{\nu} f(\lambda, \nu) \sum_{\nu_{1}, \mu_{1}} f\left(\mu_{1}, \nu_{1}\right) \delta_{\xi=-\nu+\nu_{1}} f^{-1}\left(\nu, \mu_{1}\right) f\left(\nu_{1}, \mu_{1}\right) \\
=\frac{1}{|G|} \sum_{\nu} f(\lambda, \nu) \sum_{\mu_{1}} f\left(\mu_{1}, \xi+\nu\right) f\left(\xi, \mu_{1}\right)=f^{-1}(\lambda, \xi) f^{-1}(\xi, \lambda)=\operatorname{Sym}_{G}(\hat{f})(\lambda, \xi) .
\end{aligned}
$$

This scalar factor of the double braiding is equal +1 for all $\lambda$ (and hence all $\mathbb{C}_{\psi}$ ) iff $\xi \in \operatorname{Rad}_{0}$ as asserted.

The previous two lemmas combined imply that any irreducible transparent $u_{q}(\mathfrak{g})$-module has necessarily the characters $\chi(\mu)=f(\mu, \xi), \xi \in \operatorname{Rad}_{0}$ as highest-weights, and conversely if such a character $\chi$ gives rise to 1 -dimensional $u_{q}(\mathfrak{g})$-modules (i.e., $\left.\chi\right|_{2 \Lambda_{R}}=1$ ), then these are guaranteed transparent objects. Hence the final step is to give more closed expressions for the $f$-transformed characters $\chi$ of the radical depending on the case and check the 1-dimensionality condition.

In all cases where $f$ is symmetric we have seen in Example 5.11 that $\operatorname{Rad}_{0}\left(\operatorname{Sym}_{G}(\hat{f})\right)$ is the 2-torsion subgroup of $\Lambda / \Lambda^{\prime}$, so in these cases $\chi$ gives rise to a 1-dimensional object.

Corollary 5.20. If $f$ is symmetric (true for all cases except $D_{2 n}$ ) then the transparent objects are all 1-dimensional $\mathbb{C}_{\chi}$ where the characters $\chi$ are the $f$-transformed of the elements in the radical of the bimultiplicative form $\left.\operatorname{Sym}(\hat{f})\right|_{G}$ on $G=\Lambda / \Lambda^{\prime}$. In particular the group of transparent objects is isomorphic to this radical as an abelian group.

Corollary 5.21. In the case of symmetric $f$ (all cases except $D_{2 n}$ ) the fact that $\operatorname{Rad}_{0}$ is the 2-torsion of $\Lambda / \Lambda^{\prime}$ and $f$-transformation is a group isomorphism shows:

The group $T$ of transparent objects consists of $\mathbb{C}_{\chi}$ where $\left.\chi\right|_{2 \Lambda}=1$, i.e., the two-torsion of the character group.

The remaining case in $D_{2 n}$ with $f$ nonsymmetric and has been done by hand in Lemma 5.14.

\section{Quantum groups with a ribbon structure}

In [16, Theorem 8.23], the existence of ribbon structures for $u_{q}(\mathfrak{g}, \Lambda)$ is proven. In this section we construct a ribbon structure for all cases. In the proof, we use several auxiliary results from [16].

Theorem 6.1. Let $u_{q}(\mathfrak{g}, \Lambda)$ be quasitriangular Hopf algebra, with an $R$-matrix satisfying the conditions in Theorem 2.2 and let $u:=S\left(R_{(2)}\right) R_{(1)}$. Then $v:=K_{\nu_{0}}^{-1} u$ is a ribbon element in $u_{q}(\mathfrak{g}, \Lambda)$. 
Proof. We consider the natural $\mathbb{N}_{0}\left[\alpha_{i} \mid i \in I\right]$-grading on the Borel parts $u^{ \pm}:=u_{q}(\mathfrak{g}, \Lambda)^{ \pm}[13]$. Since $u^{ \pm}$is finite-dimensional, there exists a maximal $\nu_{0} \in \mathbb{N}_{0}\left[\alpha_{i} \mid i \in I\right]$, s.t. the homogeneous component $u_{\nu_{0}}^{ \pm}$is non-trivial. More explicitly $\nu_{0}$ is of the form

$$
\nu_{0}=\sum_{\alpha \in \Phi^{+}}\left(\ell_{\alpha}-1\right) \alpha
$$

where $\ell_{\alpha}:=\frac{\ell}{\operatorname{gcd}\left(\ell, 2 d_{\alpha}\right)}$.

Using the formulas $u=\left(\sum f(\mu, \nu) K_{\mu+\nu}\right)^{-1} \vartheta$ and $S(u)=\left(\sum f(\mu, \nu) K_{\mu+\nu}\right)^{-1} S(\vartheta)$, where $\vartheta=\sum \bar{\Theta}^{(2)} S^{-1}\left(\bar{\Theta}^{(2)}\right)$, Müller proves the formula $K_{-\nu_{0}}^{2}=u^{-1} S(u)$. Using the fact that $u$ commutes with all grouplike elements, this implies $v^{2}=u S(u)$. In order to show that $v$ is central, we first show that $K_{\nu_{0}+2 \rho}^{-1}$ is a central element. By the $K, E$-relations, this is equivalent to

$$
\nu_{0}+2 \rho \in \operatorname{Cent}_{\Lambda}\left(\Lambda_{R}\right)
$$

where $\rho=\frac{1}{2} \sum_{\alpha \in \Phi^{+}} \alpha$ is the Weyl vector.

We calculate directly that this is always the case:

$$
\left(\nu_{0}+2 \rho, \beta\right)=q^{\sum_{\alpha \in \Phi^{+}}\left(\ell_{\alpha}-1+1\right)(\alpha, \beta)}=q^{\ell \sum_{\alpha \in \Phi^{+}} \frac{1}{\operatorname{gcd}\left(\ell, 2 d_{\alpha}\right)} \cdot 2 d_{\alpha}\left(\alpha^{\vee}, \beta\right)}=1 .
$$

Since $K_{2 \rho} u x=x K_{2 \rho} u$ holds for all $x \in u_{q}(\mathfrak{g}, \Lambda)$ (see [16, Lemmas 8.22 and 8.19]), we have

$$
v x=K_{\nu_{0}}^{-1} u x=K_{\nu_{0}+2 \rho}^{-1} K_{2 \rho} u x=K_{\nu_{0}+2 \rho}^{-1} x K_{2 \rho} u=x K_{\nu_{0}+2 \rho}^{-1} K_{2 \rho} u=x v,
$$

hence $v$ is central.

\section{Open questions}

Question 7.1. It was surprising to us that the case $D_{2 n}=\mathfrak{s o}_{4 n}(\mathbb{C})$ has so many more solutions that the other cases, in particular with non-symmetric $R_{0}$, due to the non-cyclic fundamental group. Do these additional modular tensor categories appear elsewhere? Does the non-symmetry have interesting implications on the category?

Question 7.2. Our procedure would be similarly possible for any diagonal Nichols algebra. The Lusztig ansatz can in these cases be found in [1].

Question 7.3. In each case where $u_{q}(\mathfrak{g}, \Lambda), R$ is not factorizable, we can modularize (see [2]) the corresponding representation category and get a modular tensor category, which should be representations over some "quasi-quantum group" $u_{q}(\mathfrak{g}, \tilde{\Lambda}, \omega), R$ which is a quasi-Hopf algebra where the group ring $\mathbb{C}[\tilde{\Lambda}]$ is deformed by a 3-group-cocycle $\omega$. Can we describe this quasi-Hopf algebra in a closed form? Moreover, is every factorizable quasi-quantum group the modularization of a quasi-triangular quantum group from our list?

More technically:

Question 7.4. The centralizer transfer map $A_{\ell}$ in Definition 3.8 (and correspondingly the form $a_{\ell}$ ) had a very general characterization, but we could only prove existence by a construction using the classification of simple Lie algebras (and distinguishing three cases). We strongly suspect that these maps exist under rather general assumptions.

Also the result Theorem 3.3 from our previous article [11] has only been proven there for cyclic groups (and by hand for $\mathbb{Z}_{2} \times \mathbb{Z}_{2}$ ) although we strongly suspect it holds for every abelian group. 


\section{Acknowledgements}

Both authors thank Christoph Schweigert for helpful discussions and support. They also thank the referees, who gave a relevant contribution to improve the article with their comments. The first author was supported by the DAAD P.R.I.M.E program funded by the German BMBF and the EU Marie Curie Actions as well as the Graduiertenkolleg RTG 1670 at the University of Hamburg. The second author was supported by the Collaborative Research Center SFB 676 at the University of Hamburg.

\section{References}

[1] Angiono I., Yamane H., The R-matrix of quantum doubles of Nichols algebras of diagonal type, J. Math. Phys. 56 (2015), 021702, 19 pages, arXiv:1304.5752.

[2] Bruguières A., Catégories prémodulaires, modularisations et invariants des variétés de dimension 3, Math. Ann. 316 (2000), 215-236.

[3] Etingof P., Gelaki S., Nikshych D., Ostrik V., Tensor categories, Mathematical Surveys and Monographs, Vol. 205, Amer. Math. Soc., Providence, RI, 2015.

[4] Feigin B.L., Gainutdinov A.M., Semikhatov A.M., Tipunin I.Yu., Modular group representations and fusion in logarithmic conformal field theories and in the quantum group center, Comm. Math. Phys. 265 (2006), 47-93, hep-th/0504093.

[5] Feigin B.L., Tipunin I.Yu., Logarithmic CFTs connected with simple Lie algebras, arXiv:1002.5047.

[6] Gainutdinov A.M., Runkel I., Symplectic fermions and a quasi-Hopf algebra structure on $\bar{U}_{q} s \ell(2)$, J. Algebra 476 (2017), 415-458, arXiv:1503.07695.

[7] Kerler T., Lyubashenko V.V., Non-semisimple topological quantum field theories for 3-manifolds with corners, Lecture Notes in Mathematics, Vol. 1765, Springer-Verlag, Berlin, 2001.

[8] Kondo H., Saito Y., Indecomposable decomposition of tensor products of modules over the restricted quantum universal enveloping algebra associated to $\mathfrak{s l}_{2}$, J. Algebra 330 (2011), 103-129, arXiv:0901.4221.

[9] Lentner S., A Frobenius homomorphism for Lusztig's quantum groups for arbitrary roots of unity, Commun. Contemp. Math. 18 (2016), 1550040, 42 pages, arXiv:1406.0865.

[10] Lentner S., Nett D., A theorem of roots of unity and a combinatorial principle, arXiv:1409.5822.

[11] Lentner S., Nett D., New R-matrices for small quantum groups, Algebr. Represent. Theory 18 (2015), 1649-1673, arXiv:1409.5824.

[12] Lusztig G., Quantum groups at roots of 1, Geom. Dedicata 35 (1990), 89-113.

[13] Lusztig G., Introduction to quantum groups, Modern Birkhäuser Classics, Birkhäuser/Springer, New York, 2010.

[14] Müller E., Quantengruppen im Einheitswurzelfall, Ph.D. Thesis, Ludwig-Maximilians-Universität München, 1998.

[15] Müller E., Some topics on Frobenius-Lusztig kernels. I, J. Algebra 206 (1998), 624-658.

[16] Müller E., Some topics on Frobenius-Lusztig kernels. II, J. Algebra 206 (1998), 659-681.

[17] Schneider H.-J., Some properties of factorizable Hopf algebras, Proc. Amer. Math. Soc. 129 (2001), 18911898.

[18] Shimizu K., Non-degeneracy conditions for braided finite tensor categories, arXiv:1602.06534.

[19] Turaev V.G., Quantum invariants of knots and 3-manifolds, De Gruyter Studies in Mathematics, Vol. 18, Walter de Gruyter \& Co., Berlin, 1994. 